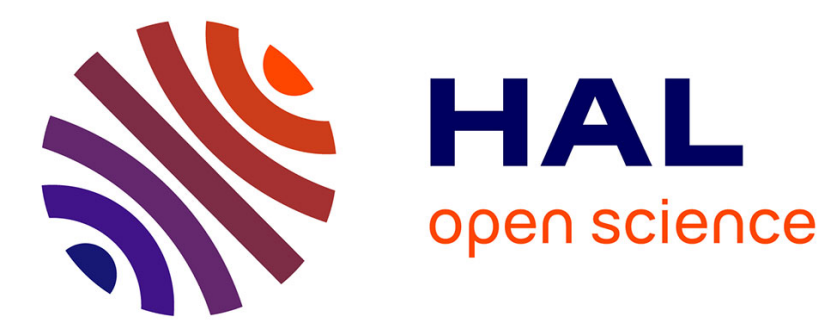

\title{
Imagerie et holographie ultrasonores
}

P. Alais

\section{To cite this version:}

P. Alais. Imagerie et holographie ultrasonores. Revue de Physique Appliquée, 1976, 11 (5), pp.559-580.

10.1051/rphysap:01976001105055900 . jpa-00244091

\section{HAL Id: jpa-00244091 \\ https://hal.science/jpa-00244091}

Submitted on 1 Jan 1976

HAL is a multi-disciplinary open access archive for the deposit and dissemination of scientific research documents, whether they are published or not. The documents may come from teaching and research institutions in France or abroad, or from public or private research centers.
L'archive ouverte pluridisciplinaire HAL, est destinée au dépôt et à la diffusion de documents scientifiques de niveau recherche, publiés ou non, émanant des établissements d'enseignement et de recherche français ou étrangers, des laboratoires publics ou privés. 


\title{
REVUE DE PHYSIQUE APPLIQUÉE
}

\author{
Supplément au * Journal de Physique *
}

\author{
Classification \\ Physics Abstracts \\ $0.610-9.782-9.820-9.840$
}

\section{IMAGERIE ET HOLOGRAPHIE ULTRASONORES}

\author{
P. ALAIS \\ Université Pierre et Marie Curie, Laboratoire de Mécanique Physique \\ 2, place de la Gare de Ceinture, 78210 Saint-Cyr l'Ecole, France
}

(Reçu le 8 mars 1976, accepté le 17 mai 1976)

\begin{abstract}
Résumé. - Cet article est une revue des nombreuses tentatives effectuées au cours des dix dernières années pour adapter au rayonnement acoustique les procédés développés en optique de Fourier et notamment l'holographie. Bien que la théorie mise en œuvre soit identique pour les deux types de rayonnements, les solutions expérimentales retenues sont très différentes du fait de l'absence de film phonosensible et de l'existence de récepteurs ultrasonores linéaires. Outre les montages classiques d'imagerie et d'holographie ultrasonore, des dispositifs de conversion d'onde acoustooptiques ont pu être développés. Plus récemment, l'intérêt manifeste de l'échographie ultrasonore dans le domaine médical a suscité le développement de techniques d'exploration rapide par le balayage et la focalisation simulés électroniquement d'un transducteur à grande ouverture.

Abstract. - This article is a review of many attempts to adapt to ultrasonics the classical Fourier Techniques of Optics over the past ten years. Though the theoretical concepts are the same, the ultrasonic techniques are quite different as there is no simple phonosensitive film and linear ultrasonic transducers are used instead. Beside classical imaging and holographic devices, several acousto-optic wave converters have been developed. More recently the growing interest of ultrasonic echography in medicine has lead to new techniques using fast electronic scanning and focusing of synthetic large apertures.
\end{abstract}

1. Imagerie et holographie ultrasonores. - 1.1 LES RAISONS ET LES BUTS. - L'utilisation d'un rayonnement autre que la lumière visible pour acquérir une information sur un domaine de l'espace où la propagation optique est impossible ou inutilisable est devenue parfaitement courante avec le radar, le sonar, la radiographie, la caméra à infra-rouge, le microscope à balayage. On notera que l'inefficacité d'un rayonnement pour délivrer une information peut résulter soit de l'opacité du domaine liée à l'atténuation, la réflexion, la diffraction ou la diffusion encourues par le rayonnement, soit au contraire de la trop grande transparence de l'espace examiné. C'est ainsi que certains organes tels que le cerveau ou le foie ne délivrent que très peu d'information à un rayonnement $X$ et en général de façon indirecte par opacification artificielle de leur vascularisation (artériographie).

On connaît depuis les premières expériences de Langevin en 1915 [1] la remarquable propagation des ultrasons dans l'eau laquelle a donné naissance aux techniques d'acoustique sous-marine à des fins mili- taires bien connues mais aussi pacifiques pour l'étude hydrographique des fonds et géologique du sous-sol marin ainsi que pour la détection des bancs de poissons. Plus récemment, l'exploitation pétrolière du plateau continental déjà entamée et celle envisagée à moyen terme des ressources en nodules métalliques des grands fonds, de même que les problèmes posés par le sauvetage de sous-marins ou l'exploration sousmarine dans des zones où l'eau est trouble, ont suscité un grand intérêt pour le développement de dispositifs d'imagerie ultrasonore à faible distance, de l'ordre de quelques dizaines de mètres, pouvant faire appel à un rayonnement ultrasonore de longueur d'onde millimétrique.

Parallèlement, depuis les années 50, une technique, progressivement devenue très fructueuse, d'échographie à courte distance calquée sur les méthodes de contrôle non destructif industriel, a permis aux médecins d'obtenir, par déplacement d'une sonde ultrasonore émettrice réceptrice, en contact direct avec le corps ou en contact aqueux (immersion), dont la ligne 
de tir est assujettie à demeurer dans un plan, des images en coupe, dites échographies $\mathrm{B}$, analogues à l'image que donne du fond le sondeur d'un bateau naviguant en ligne droite. Longtemps controversé, l'intérêt médical de ces images n'est plus à prouver et plusieurs milliers de ces appareillages fonctionnent actuellement dans les services hospitaliers notamment en obstétrique où l'irradiation $X$ est en général prohibée. On notera que, outre la différence de présentation des images, l'information ultrasonore ainsi recueillie est différente de celle que peuvent donner les rayons $X$, les critères, définissant les accidents de propagation générateurs d'information, étant évidemment très différents. C'est ainsi que la figure 1 montre à titre d'exemple qu'une

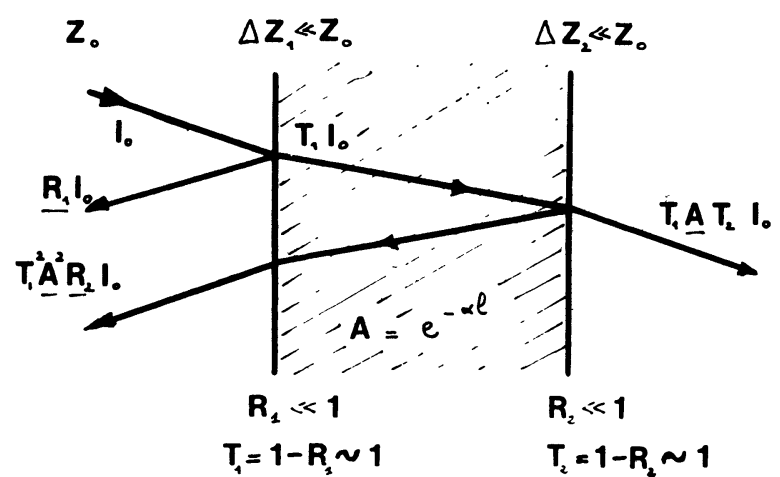

FIG. 1. - Information délivrée en échographie et en transparence par une lame semi-transparente.

lame à faces parallèles présentant une faible rupture $\Delta z$ d'impédance acoustique relativement au milieu ambiant délivre une information, liée au coefficient de réflexion, c'est-à-dire à $\Delta z$, et à l'atténuation, laquelle s'avère plus riche en échographie dans la mesure où on peut séparer les ondes réfléchies par la face avant et la face arrière. Là où les rayons $X$ ne fournissent qu'une information d'atténuation intégrée sur leur parcours quasi rectiligne du fait qu'ils échappent à la diffraction, l'information ultrasonore peut résulter autant des réflexions encourues au niveau des parois d'organes, de vaisseaux, de tumeurs ou de tissus inhomogènes à l'échelle de la longueur d'onde, que de l'atténuation. Une circonstance très favorable aux applications médicales tient à la grande uniformité tant en impédance acoustique qu'en vitesse de propagation des tissus, à l'exception bien sûr, du tissu osseux. Cette uniformité liée à la richesse en eau des tissus limite les phénomènes de réfraction de réflexion et diffraction à un taux raisonnable, permettant l'exploration en profondeur. Elle permet aussi d'espérer l'utilisation fructueuse de dispositifs d'imagerie ou d'holographie à grande ouverture dont la description est l'objet de cet article. Nous conserverons plus spécifiquement le point de vue des applications médicales bien que la plupart des considérations théoriques et techniques puissent être étendues à d'autres fins.

1.2 LE CADRE THÉORIQUE. - Le comportement acoustique des tissus organiques, très voisin en général de celui de l'eau tant en impédance qu'en célérité, permet d'adopter comme modèle simple celui de l'Acoustique ordinaire dans un liquide en équilibre hydrostatique et thermodynamique. On sait que le champ acoustique dérive alors d'un potentiel $\varphi$ selon

$$
\left.\begin{array}{ll}
\mathbf{V}=\operatorname{grad} \varphi & \text { vitesse acoustique } \\
p=-\rho_{0} \frac{\partial \varphi}{\partial t} & \text { pression acoustique }
\end{array}\right\}
$$

Les grandeurs acoustiques $p, \mathbf{V}, \rho$ la perturbation de densité acoustique et $\varphi$ obéissent alors, en l'absence de source acoustique, à l'équation d'ondes

$$
p, \rho, \varphi, \mathbf{V}=0 \text { où } \square \equiv \frac{1}{c^{2}} \frac{\partial_{2}}{\partial t^{2}}-\nabla^{2}
$$

la célérité sonique $c$ étant définie à partir de l'équation d'état adiabatique du liquide :

$$
c^{2}=(\partial p / \partial \rho)_{S}
$$

Si on admet le caractère faiblement perturbateur des inhomogénéités et de l'atténuation acoustique $(\Delta c, \Delta z$, $A)$ présentées par les tissus irradiés à un champ incident $\varphi_{0}$ on conçoit aisément qu'on puisse développer une théorie des petites perturbations où le potentiel $\varphi_{0}$ obéit à l'équation d'ondes non perturbée

$$
\square \varphi_{0}=0 \text {, équation d'ordre } 0
$$

et le potentiel $\varphi_{1}$, engendré par les inhomogénéités, à l'équation

$$
\varphi_{1}=S\left(\varphi_{0}, \Delta c, \Delta z, A\right), \text { équation d'ordre } 1 .
$$

Sans épiloguer sur ce genre de raisonnement, analogue à celui qu'on adopte en acoustique non linéaire faible, on peut noter qu'il revient à négliger comme effet du second ordre la perturbation apportée par les inhomogénéités au potentiel diffracté $\varphi_{1}$. Cette hypothèse ne pourra être confirmée véritablement que lorsque le contrôle expérimental du potentiel $\varphi_{1}$ sera valablement assuré. On sait déjà qu'elle est fausse en ce qui concerne l'atténuation moyenne subie $\operatorname{par} \varphi_{1}$ comme $\operatorname{par} \varphi_{0}$, mais ce dernier point ne soulève qu'un problème de sensibilité à la réception et n'enlève pas sa valeur à l'information délivrée. Naturellement, le caractère faiblement solide des tissus mous est aussi négligé, et les perturbations violentes que peuvent engendrer les tissus osseux d'impédance élevée, comme les poches gazeuses d'impédance quasi nulle, sont provisoirement écartées de ce modèle. Il apparaît alors que l'information acoustique souhaitée est constituée par le terme de source $S\left(\varphi_{0}, \Delta c, \Delta z, A\right)$ et qu'elle peut être acquise à l'extérieur du domaine irradié en termes du champ acoustique $\varphi_{1}$ dont elle est responsable. L'imagerie et l'holographie ultrasonores offrent un moyen plus ou moins calqué sur les procédés optiques, de remonter du champ acoustique perçu au niveau de la pupille phonosensible à sa source $S$. Le problème essentiel étant 
d'évaluer et d'interpréter, voire de contrôler l'évolution du champ acoustique véhiculant l'information hors de la zone source, on est alors amené à utiliser les considérations, tout aussi fructueuses en Acoustique, de l'Optique de Fourier qui sont à l'origine de l'holographie comme de concepts nouveaux en imagerie ordinaire et en traitement de l'information. Cette science riche d'applications nouvelles surtout depuis l'apparition de la lumière cohérente du laser n'a été véritablement développée qu'en 1946 par Duffieux [2], puis enrichie et étendue par les travaux de Maréchal [3] sur le filtrage des fréquences spatiales et pourtant elle découle de manière aussi logique que la Mécanique de ses principes fondamentaux, de la formule de Rayleigh Sommerfeld (1894) traduisant quantitativement le principe énoncé par Huygens, revu par Fresnel puis Kirchhoff. Cette formule n'est en fait qu'une conséquence mathématique de l'équation des ondes, appliquée à un rayonnement de fréquence $v$, décrit par le champ scalaire complexe

$$
\varphi(\mathbf{M}, t)=U(\mathbf{M}) \mathrm{e}^{-2 \pi j v t}
$$

et donne, en' l'absence de sources dans le demi-espace libre $z>0$ la valeur du champ $\varphi$ en tout point de ce demi-espace à partir de sa connaissance en tout point du plan $\pi_{0}(z=0)$ selon :

$U(\mathbf{M})=\frac{1}{j \lambda} \int_{\pi_{0}} U(\mathbf{P})\left(1-\frac{1}{j k P M}\right) \frac{\mathrm{e}^{j k P M}}{P M} \cos \theta \mathrm{d} S_{P}$

où $\lambda=c / v \quad$ est la longueur d'onde du rayonnement, $P \quad$ le point courant du plan $\pi_{0}\left(x_{0}, y_{0}, 0\right)$, $\mathrm{d} S_{P}=\mathrm{d} x_{0} \mathrm{~d} y_{0}$,

$k=2 \pi / \lambda$ l'amplitude du vecteur d'onde lié à la fréquence,

$\theta \quad$ l'angle de $P M$ avec la normale à $\pi_{0}$ c'est-à-dire $\mathrm{O} z$.

Une autre façon de présenter cette relation courante en optique de Fourier $[4,5]$ consiste à différentier le rôle de la variable $z$ et à traduire l'évolution du rayonnement dans le plan $\pi_{z}(z=$ Cte $)$ parallèle à $\pi_{0}$, selon $U_{z}(\mathbf{m})=U_{z}(x, y)$ en travaillant sur des fonctions de $R_{\mathrm{II}}$ paramétrées par $z$, des projections $p$ et $m$ de $P$ et $M$ sur le plan $\pi_{0}$ par exemple, ce qui donne :

$$
U_{z}(\mathbf{m})=\int U_{0}(\mathbf{p}) h_{z}(\mathbf{p m}) \mathrm{d} S_{P}
$$

notant

$h_{z}(\mathbf{p m})=\frac{1}{j \lambda} \frac{z \mathrm{e}^{j k\left((p m)^{2}+z^{2}\right)^{1 / 2}}}{(p m)^{2}+z^{2}}\left(1-\frac{1}{j k\left((p m)^{2}+z^{2}\right)^{1 / 2}}\right)$.

Cette relation de convolution dans $R_{\mathrm{II}}$ :

$$
U_{z}=U_{0} * h_{z}
$$

apparaît en fait beaucoup plus simplement dans l'espace de Fourier des fréquences spatiales $f_{x}, f_{y}$, associées aux variables $x, y$. Si on note en effet,

$$
A_{0}\left(f_{x}, f_{y}\right)=\int U_{0}(x, y) \mathrm{e}^{-2 j \pi\left(f_{x} x+f_{y} y\right)} \mathrm{d} x \mathrm{~d} y,
$$

la transformée de Fourier dans $R_{\mathrm{II}}$ de $U_{0}$, on sait qu'on peut interpréter la relation inverse,

$$
U_{0}(\mathbf{m})=\int A_{0}(\mathbf{f}) \mathrm{e}^{2 j \pi \mathrm{f}, \mathrm{m}} \mathrm{d} \sigma_{f}, \quad \mathrm{~d} \sigma_{f}=\mathrm{d} f_{x} \mathrm{~d} f_{y},
$$

comme une décomposition du rayonnement étudié observé au niveau du plan en ondes planes se propageant vers les $z>0$. L'onde plane de fréquence $v$ (ou pulsation $\omega$ ) se propageant dans la direction $\mathbf{n}(\alpha, \beta, \gamma)$ de vecteur d'onde $\mathbf{k}=k \mathbf{n}(k=2 \pi v / c)$ de coordonnées :

$$
\begin{aligned}
& k_{x}=2 \pi f_{x}, \quad k_{y}=2 \pi f_{y}, \\
& k_{z}=2 \pi \sqrt{v^{2} / c^{2}-f_{x}^{2}-f_{y}^{2}},
\end{aligned}
$$

est représentée par le facteur de phase spatiale $\mathrm{e}^{j\left(k_{x} x+k_{y} y+k_{z} z\right)}$ qui a bien au niveau du plan $\pi_{0}$ la signature $e^{2 j \pi f . m}$ apparaissant dans la relation (10), laquelle peut alors être réécrite selon

$$
\varphi(\mathbf{M}, t)=\int A_{0}(\mathbf{f}) \mathrm{e}^{j(\mathbf{k} . \mathbf{r}-\omega t)} \mathrm{d} \sigma_{f} .
$$

On voit que le caractère réel de $k_{z}>0$ limite le domaine de $\mathbf{f}$ au cercle $f<v / c$. Dans cette nouvelle écriture, la relation (8) s'écrit dans l'espace de Fourier $\left(f_{x}, f_{y}\right)$ selon

$$
A_{z}=A_{0} H_{z}
$$

définissant les transformées de Fourier $A_{z}$ et $H_{z}$ de $U_{z}$ et $h_{z}$ comme en (9) pour $U_{0}$. Naturellement la relation (10) permet d'identifier sans démonstration la transformée $H_{z}$ au facteur de phase :

$$
H_{z}\left(f_{x}, f_{y}\right)=\mathrm{e}^{j k_{z} z}=\exp \left(2 j \pi_{z} \sqrt{v^{2} / c^{2}-f_{x}^{2}-f_{y}^{2}}\right)
$$

affectant chacune des signatures des composantes planes du rayonnement considéré quand on passe du plan $\pi_{0}$ au plan $\pi_{z}$.

La relation de Rayleigh Sommerfeld (5) et ses nouvelles formes (8) et (11) sont rigoureuses pour un rayonnement illimité dans le demi-espace $z>0$ et en l'absence de sources dans ce domaine. En réalité, le champ est physiquement limité et modifié au niveau de certains plans $\pi_{z}$ par des diaphragmes, des lentilles ou des hologrammes. Nous admettrons par la suite qu'une telle opération ne perturbe pas le champ incident et se traduit mathématiquement par le découpage (et la modulation éventuelle),

$$
U_{z+}=U_{z-} P_{z}
$$

où la fonction pupille $P_{z}$ est nulle pour les points extérieurs à la surface de la pupille. Elle est égale à 1 pour les points intérieurs s'il s'agit d'un simple diaphragme 
mais pourra être aussi une fonction de transparence $t(x, y)$ en holographie ou encore un facteur de déphasage s'il s'agit d'une lentille mince. On notera qu'un tel découpage peut induire dans la transformée de Fourier

$$
A_{z+}=A_{z-} * \pi_{z} \quad \text { où } \quad \pi_{z}\left(f_{x}, f_{y}\right)=T F\left(P_{z}\right)
$$

des composantes spectrales de fréquence $f>v / c$, évanescentes en $z$, dont l'effet disparaît dans une propagation supérieure à quelques longueurs d'ondes, ce qui justifie en général l'emploi sans précautions particulières de $U_{z+}$.

2.1 Principe DE L'HOlographe. - En affirmant que la connaissance du rayonnement $\varphi$ au niveau du plan $\pi_{0}(z=0)$ entraîne sa connaissance dans le demiespace $z>0$ dans les conditions précisées plus haut, le principe d'Huygens et sa traduction précise par la relation de Rayleigh Sommerfeld, impliquent que la création d'un rayonnement $\varphi$ n'exige que son imposition correcte au niveau du plan $\pi_{0}$ par exemple. L'holographie ou méthode de reconstruction d'onde proposée par D. Gabor en 1948 [6], constitue un moyen simple et élégant d'imposer les bonnes conditions aux limites requises à la variable caractérisant le rayonnement. Dans'une première phase (Fig. $2 a$ ), on utilise un détecteur quadratique sensible à l'énergie incidente pour relever la figure d'interférences obtenue en superposant au champ $\varphi$ étudié le rayonnement $\varphi_{0}$ émis par une source ponctuelle 0 en cohérence de phase avec $\varphi$. De telles conditions sont réunies lorsqu'on utilise un laser pour éclairer la cible diffractante réémettant le rayonnement $\varphi$ et une lame semi-transparente pour prélever une part du rayonnement laser pour créer le fond cohérent $\varphi_{0}$. Une plaque photosensible convenablement exposée fournit une figure de transparence variant approximativement, dans une plage convenable comme l'énergie reçue soit
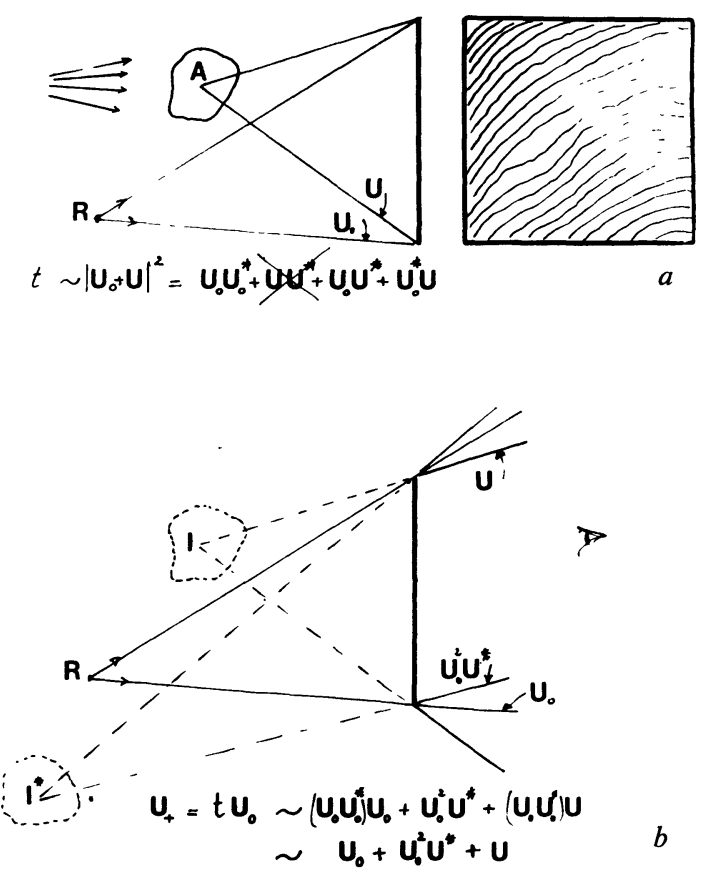

Fig. 2. - a) Enregistrement holographique. b) Restitution holographique.

$$
\begin{array}{r}
t(x, y) \sim\left|\varphi_{0}+\varphi\right|^{2}=\left(U_{0}+U\right)\left(U_{0}+U\right)^{*}= \\
=U_{0} U_{0}^{*}+U_{0} U^{*}+U U_{0}^{*}+U U^{*}
\end{array}
$$

La figure d'interférences ainsi obtenue, dite hologramme, permet de garder en mémoire non une image mais l'onde $\varphi$ émise par l'objet diffractant. En effet, si dans une seconde phase, dite de restitution holographique (Fig. 2b), on éclaire l'hologramme dans la même position avec la même source 0 , le rayonnement $\varphi_{0}\left(U_{0}\right)$ se trouve modulé par la transparence $t(x, y)$ selon :

$$
\begin{aligned}
U_{+}=t U_{0} & \sim U_{0}\left(U_{0} U_{0}^{*}\right)+U_{0}^{2} U_{+}^{*}+\left(U_{0} U_{0}^{*}\right) U+U_{0}\left(U U^{*}\right) \\
& =U_{1}+U_{2}+U_{3}+U_{4} .
\end{aligned}
$$

Du fait de la linéarité du principe d'Huygens on peut dire que chacun des termes donne naissance à une onde particulière à droite de l'hologramme. L'onde 1 ne fait que prolonger l'onde $U_{0}$ faiblement modulée par les variations qu'on peut négliger de l'éclairement $\left(U_{0} U_{0}^{*}\right)$. L'onde 3 restitue le rayonnement étudié $U$ avec la même distorsion faible. L'onde 4 peut être négligée si on a pris la précaution d'utiliser un fond cohérent suffisamment intense en regard de l'onde étudiée $\left(\left|U_{0}\right| \gg|U|\right)$. Enfin le terme 2 est responsable d'une onde porteuse d'information comme l'onde 3 . Naturellement l'observation de l'onde 3 reconstruite, avec des moyens usuels (œil, caméra, appareil photographique), permet d'obtenir des images de l'objet comme s'il était réellement présent avec la possibilité de l'observer sous différents angles avec des mises au point différentes, etc... ce qui justifie l'appellation de reconstruction holographique tridimensionnelle. La simplicité de l'enregistrement holographique qui n'utilise qu'un champ scalaire $t(x, y)$ pour restituer un champ complexe $U$ se paie par la superposition à l'onde reconstruite des rayonnements $U_{1}, U_{2}$ et $U_{4}$. Si l'onde $U_{4}$ peut en général, comme il a été dit, être négligée, il faut prendre des précautions pour éviter la gêne ou l'ambiguïté apportée par les termes $U_{1}$ et $U_{2}$, ce dernier donnant en particulier une autre image de l'objet dite image conjuguée dont on doit éviter la superposition à celle qu'on souhaite obtenir. L'expérience décrite plus haut utilise le même éclairement à l'enregistrement et à la restitution ce qui simplifie considérablement l'interprétation des résultats obtenus. En pratique, il n'est pas nécessaire en holographie 
optique d'utiliser la même source à l'enregistrement et à la restitution. Par ailleurs, l'holographie acoustique consiste à effectuer un enregistrement holographique à l'aide d'un rayonnement acoustique et à opérer la reconstitution en lumière cohérente visible afin de substituer à l'onde acoustique diffractée étudiée une onde optique permettant l'observation directe de l'objet. Il y a alors des modifications de géométrie quant à la disposition. des sources, aux dimensions de l'hologramme, avec changement de la longueur d'onde utilisée qui requièrent l'utilisation de formules de stigmatisme holographique analogues à celles qu'on connaît grâce à l'optique géométrique pour les lentilles et qu'il nous appartient de développer maintenant dans le cadre de l'approximation de Fresnel.

2.2 L'APPROXIMATION DE Fresnel. - Une simplification considérable intervient dans l'emploi de la formule de Rayleigh Sommerfeld grâce à l'approximation de Fresnel laquelle consiste à remarquer que la géométrie des dispositifs d'imagerie ou d'holographie est telle que dans l'expression (7) de $h(\mathbf{p m})$, on peut tenir compte de ce que $\delta=$ pm reste en général plus petit en module que la distance $z$ des plans $\pi_{0}$ et $\pi_{z}$ du fait de la limitation des ouvertures utilisées au niveau de ces plans. On peut alors développer $P M$ selon :

$$
P M=z+\frac{1}{2} \frac{\delta^{2}}{z}+\frac{1}{8} \frac{\delta^{4}}{z^{3}}+\cdots
$$

et ne retenir que les deux premiers termes dans l'expression de la phase ce qui conduit à une erreur raisonnable autant que :

$$
k \frac{\delta^{4}}{8 z^{3}} \ll 2 \pi \quad \text { soit } \quad z^{3} \gg \frac{\pi \delta^{4}}{4 \lambda} .
$$

On peut d'autre part négliger sans scrupule $(k P M)^{-1}$ devant 1 et conserver l'approximation plus grossière mais suffisante pour l'amplitude $\cos \theta / P M \sim 1 / z$, ce qui fournit l'expression simplifiée de $h(p \mathbf{m})$ :

$$
h(\mathbf{p m})=\frac{\mathrm{e}^{j k_{z}}}{j \lambda z} \exp \left(j \Pi \frac{(p m)^{2}}{\lambda z}\right) .
$$

Une remarque, fort utile pour l'élaboration des formules de stigmatisme, consiste par ailleurs à reconnaître au niveau du plan $\pi_{0}$, en inversant la correspondance dans le sens $\pi_{z} \rightarrow \pi_{0}$, la signature en phase de l'onde sphérique divergente émise par la source ponctuelle en amont de $\pi_{0}$ à la distance $M H(z=\overrightarrow{H M}<0)$ (ou d'une onde sphérique convergeant vers l'image ponctuelle $M$ en aval de $\pi_{0}, z>0$ ) selon la loi de phase :

$$
\exp \left(-j \pi \frac{(p m)^{2}}{\lambda z}\right)
$$

caractérisée par des cercles en phase, centrés sur $m$, dont les carrés des rayons croissent selon une progression arithmétique comme la figure des anneaux de Newton bien connue.
2.3 Approximation paraxiale. - Un calcul élémentaire montre que dans l'approximation de Fresnel une lentille mince constituée d'un milieu d'indice $n$ relatif au milieu propagatif c'est-à-dire de célérité $C^{\prime}=C / n$, limité par deux parois sphériques de faible courbure, se comporte essentiellement comme un dispositif déphaseur revenant à effectuer, à un déphasage près, constant donc sans importance, l'opération suivante sur le rayonnement incident (Fig. 3),

$$
U_{+}=L U_{-} \quad \text { où } L=\exp \left(-j \pi \frac{p^{2}}{\lambda d_{f}}\right)
$$

où $d_{f}$ est la distance focale de la lentille.

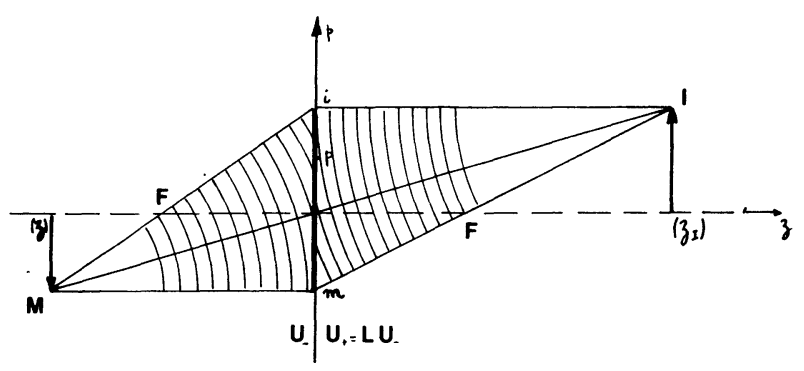

Fig. 3. - Stigmatisme des lentilles.

Dans ces conditions, la signature de l'onde incidente émise par l'objet ponctuel $M$ se trouve modifiée selon

$$
U_{+} \sim \exp \left(-j \pi \frac{(p m)^{2}}{\lambda z}\right) \cdot \exp \left(-j \pi \frac{p^{2}}{\lambda d_{f}}\right)
$$

laquelle s'identifie à la signature de l'onde associée à l'image $I$ à un déphasage constant près indépendant de $\mathbf{p}$ en notant les coefficients des termes quadratiques et linéaires en $\mathbf{p}$ :

$$
\begin{aligned}
& \frac{1}{\lambda z_{\mathrm{I}}}=\frac{1}{\lambda z}+\frac{1}{\lambda d_{f}} \\
& \frac{\mathbf{i}}{\lambda z_{\mathbf{I}}}=\frac{\mathbf{m}}{\lambda z} \\
& \left.U_{+} \sim \exp \left(-j \pi \frac{(p i)^{2}}{\lambda z_{\mathrm{I}}}\right)\right\} .
\end{aligned}
$$

D'où leś relations de correspondance objet-image bien connues :

$$
\frac{1}{z_{\mathrm{I}}}-\frac{1}{z}=\frac{1}{d_{f}}, \quad \mathbf{i}=\frac{z_{\mathrm{I}}}{z} \mathbf{m} .
$$

2.4 APPROXIMATION PARAXIALE DES SYSTÈMES HOLOGRAPHIQUES. - Le raisonnement précédent s'étend sans difficultés à l'holographie.

L'enregistrement holographique est effectué à l'aide d'un rayonnement (acoustique par exemple) de longueur d'onde $\lambda_{\mathrm{A}}$. L'onde de référence émise par la source ponctuelle A et l'onde diffractée par le point courant $M$ de l'objet s'écrivent respectivement au niveau de la pupille holographique $\pi_{\mathbf{A}}$ :

$$
U_{\mathrm{A}} \sim \exp \left(-j \pi \frac{(a p)^{2}}{\lambda_{\mathrm{A}} z_{\mathrm{A}}}\right), \quad U \sim \exp \left(-j \pi \frac{(m p)^{2}}{\lambda_{\mathrm{A}} z}\right),
$$


et la transparence holographique enregistrée :

$$
\begin{aligned}
t \sim\left(U_{\mathrm{A}}+U\right)\left(U_{\mathrm{A}}+U\right)^{*}= \\
\quad=U_{\mathrm{A}} U_{\mathrm{A}}^{*}+U_{\mathrm{A}} U^{*}+U U_{\mathrm{A}}^{*}+U U^{*} .
\end{aligned}
$$

En pratique, l'hologramme est réduit pour la restitution optique dans un rapport $\varepsilon$ ce qui fait que, dans les nouvelles coordonnées de la pupille holographique optique $\pi_{0}, \mathbf{p}^{\prime}=\varepsilon \mathbf{p}$, les termes de transparence associés aux produits $U U_{\mathrm{A}}^{*}$ et $U_{\mathrm{A}} U^{*}$ dont on sait qu'ils sont responsables de la restitution de l'onde $U$ et de l'onde conjuguée s'écrivent :

$$
t_{\mathrm{I}, \mathrm{I}^{*}} \sim \exp \left[\mp j \frac{\pi}{\varepsilon^{2}}\left(\frac{\left(m^{\prime} p^{\prime}\right)^{2}}{\lambda_{\mathrm{A}} z}-\frac{\left(a^{\prime} p^{\prime}\right)^{2}}{\lambda_{\mathrm{A}} z_{\mathrm{A}}}\right)\right] .
$$

La restitution optique consiste simplement à éclairer la pupille holographique de transparence $t^{\prime}$ à l'aide d'une source ponctuelle optique 0 de longueur d'onde $\lambda_{0}$. Cette source délivre au niveau de la pupille le rayonnement

$$
U_{0} \sim \exp \left(-j \pi \frac{\left(o p^{\prime}\right)^{2}}{\lambda_{0} z_{0}}\right)
$$

lequel se trouve modulé, après traversée de l'hologramme, du fait des termes $t_{\mathrm{I}}$ et $t_{\mathrm{I}}{ }^{*}$ selon

$$
\begin{aligned}
t_{\mathrm{I}, \mathrm{I}^{*}} U_{0} & =U_{\mathrm{I}, \mathrm{I}^{*}} \sim \exp \times \\
& \times\left\{-j \pi\left[\frac{\left(o p^{\prime}\right)^{2}}{\lambda_{0} z_{0}} \pm \frac{1}{\varepsilon^{2}}\left(\frac{\left(m^{\prime} p^{\prime}\right)^{2}}{\lambda_{\mathrm{A}} z}-\frac{\left(a^{\prime} p^{\prime}\right)^{2}}{\lambda_{\mathrm{A}} z_{\mathrm{A}}}\right)\right]\right\} .
\end{aligned}
$$

Comme dans le cas des lentilles minces on vérifie alors que les deux signatures ainsi obtenues correspondent à deux ondes sphériques liées à l'image $\mathrm{I}$ du point $M$ réelle $\left(z_{\mathrm{I}}>0\right)$ ou virtuelle $\left(z_{\mathrm{I}}<0\right)$ et à son image conjuguée $I^{*}$. Les formules de correspondance objetimages s'écrivent par identification des termes quadratiques et linéaires en $\mathbf{p}^{\prime}$ :

$$
\left.\begin{array}{r}
\frac{1}{\lambda_{0}}\left(\frac{1}{z_{\mathrm{I}}}-\frac{1}{z_{0}}\right)= \pm \frac{1}{\varepsilon^{2} \lambda_{\mathrm{A}}}\left(\frac{1}{z}-\frac{1}{z_{\mathrm{A}}}\right) \\
\frac{1}{\lambda_{0}}\left(\frac{\mathbf{i}}{z_{\mathrm{I}}}-\frac{\mathbf{o}}{z_{0}}\right)= \pm \frac{1}{\varepsilon^{2} \lambda_{\mathrm{A}}}\left(\frac{\mathbf{m}^{\prime}}{z}-\frac{\mathbf{a}^{\prime}}{z_{\mathrm{A}}}\right)= \\
= \pm \frac{1}{\varepsilon \lambda_{\mathrm{A}}}\left(\frac{\mathbf{m}}{z}-\frac{\mathbf{a}}{z_{\mathrm{A}}}\right)
\end{array}\right\}
$$

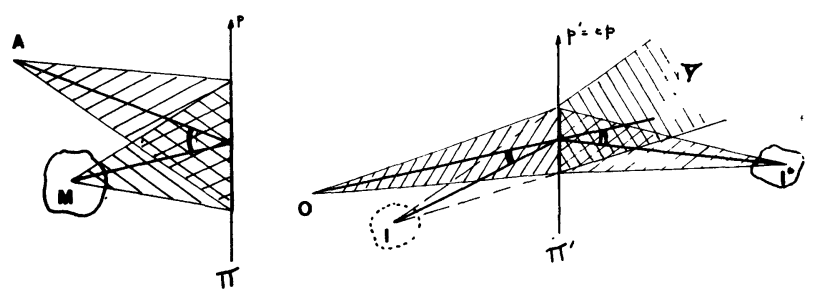

FIG. 4. -- Stigmatisme holographique.
Ces résultats appellent quelques commentaires :

La correspondance ainsi précisée n'est valable qu'autant que les sources de référence $(\mathrm{A})$ et des restitutions $(0)$ sont véritablement ponctuelles.

Elle s'effectue d'un plan-objet $\pi_{M}(z=$ Cte $)$ au plan image $\pi_{\mathrm{I}, \mathrm{I}^{*}}$ selon le grandissement axial :

$$
\frac{\mathrm{d} z_{\mathrm{I}}}{\mathrm{d} z}= \pm \frac{\lambda_{0}}{\varepsilon^{2} \lambda_{\mathrm{A}}}\left(\frac{z_{\mathrm{I}}}{z}\right)^{2}
$$

et selon le grandissement transversal :

$$
\frac{\mathrm{d} \mathbf{i}}{\mathrm{d} \mathbf{m}}= \pm \frac{\lambda_{0}}{\varepsilon \lambda_{\mathrm{A}}} \frac{z_{\mathrm{I}}}{z}
$$

la localisation angulaire des images est imposée $\operatorname{par}(\operatorname{tg} \theta \sim \theta)$

$$
\theta_{\mathrm{I}}-\theta_{0}= \pm \frac{\lambda_{0}}{\varepsilon \lambda_{\mathrm{A}}}\left(\theta_{\mathrm{M}}-\theta_{\mathrm{A}}\right)
$$

ce qui signifie que les images directe et conjuguée sont localisées angulairement symétriquement par rapport à la source de restitution.

Le grandissement angulaire associé s'écrit

$$
\frac{\mathrm{d} \theta_{\mathrm{I}}}{\mathrm{d} \theta_{\mathrm{M}}}= \pm \frac{\lambda_{0}}{\varepsilon \lambda_{\mathrm{A}}}
$$

Dans le cas particulier où les sour ces de référence $(\mathrm{A})$ et de restitution $(0)$ sont rejetées à l'infini normalement à la pupille les formules se réduisent à :

$$
z_{\mathrm{I}}= \pm \varepsilon^{2} \frac{\lambda_{\mathrm{A}}}{\lambda_{0}} z, \quad \mathbf{i}=\mathbf{m}^{\prime}=\varepsilon \mathbf{m} .
$$

Les deux images I et I* sont restituées symétriquement par rapport au plan de la pupille holographique avec une distorsion longitudinale $\varepsilon \lambda_{\mathrm{A}} / \lambda_{0}$ en dilatant fortement en général l'objet dans la direction normale à la pupille sauf si la réduction $\varepsilon$ est suffisante pour compenser le rapport $\lambda_{\mathrm{A}} / \lambda_{0} \gg 1$. Dans ce dernier cas, les images optiques de l'objet restituent ce dernier selon une réduction homothétique dans le rapport $\lambda_{0} / \lambda_{\mathrm{A}}$ et astreignent l'observateur à utiliser un instrument d'optique à fort grossissement et faible profondeur de champ, ce qui fait perdre en partie à l'holographie acoustique les avantages de la restitution tridimensionnelle opérée en holographie optique.

2.5 PouvolR DE RÉSOLUTION. - Le raisonnement ayant permis l'élaboration de la correspondance objetimage en imagerie ordinaire holographique ne tient pas compte de l'ouverture limitée en pratique de la lentille ou de la pupille holographique. Les effets parasites induits par le découpage de l'onde émergente par la fonction pupille $P(\mathbf{p})(=1$ si $\mathbf{p} \in$ pupille, $=0$ si p extérieur) sont d'autant plus réduits que les dimensions de la pupille sont plus grandes ou corrélativement la longueur d'onde du rayonnement plus petite. Si, continuant de négliger provisoirement ces effets, on poursuit l'identification de la signature de Fresnel de 
l'onde émergeant de la pupille, associée non à un point $M$ mais au rayonnement $U_{z}(\mathrm{~m})$ dans le plan $\pi_{z}$, un calcul élémentaire montre que l'onde-image $U_{z_{I}}\left(m_{I}\right)$ définie dans le plan $\pi_{z_{I}}$ associé à $\pi_{z}$ dans la correspondance objet-image peut s'obtenir à partir de la relation :

$$
U_{z_{1}}[\mathrm{i}(\mathrm{m})]=A U_{\mathrm{z}}(\mathrm{m}) \exp \left[\mathrm{j} \pi\left(\frac{i^{2}}{\lambda_{0} z_{\mathrm{I}}}=\frac{m^{2}}{\lambda_{\mathrm{A}} z}\right)\right]
$$

où $A$ est une constante complexe liée au grandissement obtenu aux caractéristiques des sources de référence acoustique et optique s'il s'agit d'holographie, du film holographique, etc... Elle reflète donc fidèlement le rayonnement objet à une distorsion de phase près qui est sans importance tant qu'on s'intéresse finalement à une image d'intensité liée seulement à $|U|^{2}$.

$\mathrm{Si}$ on note désormais $U_{\mathrm{I}}(\mathrm{i})$ cette image idéale, un raisonnement classique (Annexe I) permet de traduire les effets induits par le découpage de l'onde émergente selon la fonction pupille $P(\mathbf{p})$ en écrivant :

$$
U_{z \mathbf{I}}\left(\mathbf{m}_{\mathrm{I}}\right)=U_{\mathrm{I}}\left(\mathbf{m}_{\mathrm{I}}\right) * h\left(\mathbf{m}_{\mathrm{I}}\right)
$$

relation qui exprime que l'image réelle est obtenue en convolant l'image idéale à la fonction $h$ définie à partir de la transformée de Fourier $A_{\mathrm{P}}(\mathbf{f})$ de la fonction pupille selon :

$$
h\left(\mathrm{im}_{\mathrm{I}}\right)=\frac{1}{\lambda_{0}^{2} z_{\mathrm{I}}^{2}} A_{\mathrm{P}}\left(\frac{\mathbf{i m}_{\mathrm{I}}^{+}}{\lambda_{0} z_{\mathrm{I}}}\right) .
$$

La fonction $h$ représente l'image d'une source ponctuelle dans $\pi_{z}$, laquelle apparaît étalée selon une tache d'autant plus grande que la pupille est plus petite. A titre d'exemple, à une pupille circulaire de rayon $a$ correspond la transformée de Fourier :

$$
A_{\mathrm{P}}(\mathbf{f})=a^{2} \frac{J_{1}(2 \pi a f)}{a f}
$$

dont le premier zéro correspond à $a f=0,61$, ce qui fournit le rayon $r$ de la tache centrale

$a \frac{r_{0}}{\lambda_{0} z_{\mathrm{I}}}=0,61, \quad r_{0}=0,61 \frac{\lambda_{0} z_{\mathrm{I}}}{a}=1,22 \frac{\lambda_{0} z_{\mathrm{I}}}{D}$.

Naturellement deux points ne pourront fournir d'images distinctes que si les deux taches associées n'empiètent pas trop l'une sur l'autre. Sans entrer dans des détails qui dépassent le but qu'on s'est fixé ici, il faut cependant noter que le critère de Rayleigh qui assigne à la distance de deux points (évaluée dans le plan-image $\pi_{z_{\mathrm{I}}}$ ) pour qu'on puisse les séparer, une valeur minimale égale à $r_{0}$ dans le cas de la pupille circulaire n'est valable qu'en imagerie incohérente, la distance de résolution ainsi définie s'avérant un peu insuffisante en imagerie cohérente si les deux points émettent en phase. On montre en effet que dans le cas habituel de l'imagerie monochromatique incohérente la formation de l'image procède par sommation des intensités liées à chaque point de l'objet en sorte que l'intensité image résultante s'écrit :

$$
I_{z_{\mathrm{I}}}\left(\mathbf{m}_{\mathrm{I}}\right)=U_{\mathrm{I}} U_{\mathrm{I}}^{*} * h h^{*} .
$$

Or, la sommation des taches en intensité est en général plus favorable à la formation des images que la sommation en amplitude décrite par (27) rencontrée en imagerie cohérente laquelle est souvent responsable de l'apparition de franges ou de granulations parasites désignées sous le nom d'effet Speckle lesquelles peuvent prendre un aspect parfois très gênant en holographie acoustique.

Une autre manière fructueuse d'apprécier la limitation induite dans la résolution du dispositif utilisé du fait des dimensions de la pupille consiste à écrire la formule transposée de la relation (27) dans l'espace de Fourier des fréquences spatiales soit, notant $A_{z_{1}}, A_{1}$ et $H$ les transformées de Fourier de $U_{z_{\mathbf{I}}}, U_{\mathrm{I}}$ et $h$

$$
A_{z_{\mathrm{I}}}(\mathbf{f})=A_{\mathrm{I}}(\mathbf{f}) H(\mathbf{f})
$$

ce qui permet d'interpréter l'effet de la pupille comme un filtrage des fréquences spatiales du rayonnement induit par l'objet. L'étendue du spectre objet déjà limitée, on le sait, au niveau de l'onde diffractée au domaine $\left(f<1 / \lambda_{\mathrm{A}}\right)$ et au niveau de l'onde restituée à $\left(f<1 / \lambda_{0}\right)$ se trouve réduite par le facteur :

$$
\begin{aligned}
H(\mathbf{f}) & =T F\left[\frac{1}{\lambda_{0}^{2} z_{\mathrm{I}}^{2}} A_{\mathrm{P}}\left(\frac{\mathrm{m}_{\mathrm{I}}}{\lambda_{0} z_{\mathrm{I}}}\right)\right], \quad\left(A_{\mathrm{P}}=T F(P(\mathbf{p}))\right) \\
& =P\left(-\lambda_{0} z_{\mathrm{I}} \mathbf{f}\right)
\end{aligned}
$$

c'est-à-dire dans le cas de la pupille circulaire de rayon $a$ au domaine $f<a / \lambda_{0} z_{\mathrm{I}}$.

On voit que dans tous les cas la réduction d'information spectrale ou la distance de résolution sont directement liées à un facteur numérique près voisin de 1 , à la longueur caractéristique $L=\lambda_{0} z_{\mathrm{I}} / D^{\prime}=\lambda_{0} / \theta$ où $\theta=D^{\prime} / z_{\mathrm{I}}$, quotient d'un diamètre caractéristique de la pupille à la distance du plan image caractérise l'ouverture angulaire utilisée.

Naturellement ces résultats exprimés en termes du rayonnement émergent et dans le plan image peuvent s'énoncer de manière tout à fait comparable en termes du rayonnement diffracté par l'objet et dans le plan objet en faisant apparaître l'ouverture angulaire amont $\theta=D / z$, où $D$ représente par exemple le diamètre caractéristique de la pupille d'enregistrement holographique. La distance de résolution dans le plan objet caractérise alors directement le pouvoir de résolution du dispositif utilisé. C'est ce qui justifie l'emploi en microscopie d'objectifs à grande ouverture angulaire. L'imagerie acoustique est aussi un exemple où il est souhaitable de travailler à grande ouverture, les détails à observer n'étant pas très grands en général devant la longueur d'onde du rayonnement utilisé. On peut noter aussi que les rebondissements de la fonction $h$ définie en (26) s'expliquent du fait que le filtrage spatial opéré par la pupille s'effectue selon un découpage brutal du 
domaine des fréquences spatiales. On peut notablement amortir ce phénomène en utilisant une pupille progressivement atténuatrice procédé qui peut s'avérer intéressant dans la réalisation de lentilles acoustiques ou d'expériences holographiques.

2.6 ECHANTILlONNAGE ET AMBIGUïTÉ. - Un problème propre aux techniques d'holographie acoustique développées à l'aide du balayage d'un transducteur ou de réseaux matriciels résulte du fait que l'enregistrement n'est pas effectué continûment sur la rétine holographique mais de manière discrète. Si on note

$$
\operatorname{Pgn}(x)=\sum_{-\infty}^{+\infty} \delta(x-n),
$$

(fonction peigne, $n$ entier), un échantillonnage, à base carrée de pas $\delta$, de l'onde incidente à l'intérieur de la pupille $P$ s'écrira (toujours en termes de l'échelle de restitution, avec le rayonnement émergent) :

$$
\begin{gathered}
U_{+}=U_{-} P(\mathbf{p}) \operatorname{Pgn}\left(\frac{\mathbf{p}}{\delta}\right), \\
\operatorname{Pgn}\left(\frac{\mathbf{p}}{\delta}\right)=\operatorname{Pgn}\left(\frac{x}{\delta}\right) \cdot \operatorname{Pgn}\left(\frac{y}{\delta}\right)
\end{gathered}
$$

et notant

$$
P^{\prime}(\mathbf{p})=P(\mathbf{p}) \operatorname{Pgn}\left(\frac{\mathbf{p}}{\delta}\right) .
$$

On est conduit à réécrire $\left(25^{\prime}\right)$ à partir de la nouvelle transformée de $P^{\prime}$ :

$$
A_{\mathrm{P}^{\prime}}(\mathbf{f})=A_{\mathrm{P}}(\mathbf{f}) * \delta^{2} \operatorname{Pgn}(\delta \mathbf{f})
$$

laquelle se trouve constituée très simplement par la somme de la transformée $A_{\mathrm{p}}(\mathrm{f})$ et des termes déduits de $A_{\mathrm{P}}(\mathbf{f})$ par la translation dans l'espace des f selon

$$
A_{\mathbf{P}^{\prime}}(\mathbf{f})=\sum_{\mathbf{n}} A_{\mathbf{P}}\left(\mathbf{f}-\frac{\mathbf{n}}{\delta}\right), \quad \mathbf{n}\left\{\frac{n_{x}}{n_{y}} \text { entiers } \gtrless 0 .\right.
$$

Dans ces conditions, la tache $h\left(\mathbf{i m}_{\mathrm{I}}\right)$ définie en (26), associée à une source ponctuelle, n'est plus seulement distribuée au voisinage de $\mathbf{i}$ mais de tous les points du réseau $\mathbf{i}+L \mathbf{n}$ où $L=\lambda_{0} z_{\mathrm{I}} / \delta$ et un calcul élémentaire montre qu'il faut alors corriger les formules (26) et (27) en tenant compte du terme de phase lié à $\left(m_{\mathrm{I}}^{2}-i^{2}\right)$ selon

$$
\begin{aligned}
& h^{\prime}(\mathbf{m})=h(\mathbf{m}) * \operatorname{Pgn}\left(\frac{\mathbf{m}}{L}\right)=\sum_{\mathbf{n}} h(\mathbf{m}-L \mathbf{n}), \\
& U_{z_{\mathbf{I}}}^{\prime}(\mathbf{m})=\sum_{\mathbf{n}} U_{\mathrm{I}}(\mathbf{m}) * \exp \left(2 j \pi \frac{L \mathbf{n} \cdot \mathbf{m}}{\lambda_{0} z_{\mathrm{I}}}\right) h(\mathbf{m}-L \mathbf{n}) .
\end{aligned}
$$

L'échantillonnage a donc pour conséquence de créer un réseau d'images centrées sur les directions de Bragg associées au réseau diffractant que constitue la pupille échantillonneuse.

Pour lever toute ambiguïté dans l'information ainsi fournie, il est indispensable de séparer complètement ces images. En principe, si la dimension caractéristique de l'image est $\Delta$ il suffit d'imposer au pas d'échantillonnage une valeur qui satisfasse à :

$$
L=\lambda_{0} z_{\mathrm{l}} / \delta>\Delta, \text { soit } \delta<\lambda_{0} z_{\mathrm{I}} / \Delta .
$$

C'est en fait la condition qui doit être satisfaite si on effectue par exemple la reconstruction de l'onde par le calcul à partir des données complexes $U_{i j}=U\left(x_{i}, x_{j}\right)$ échantillonnées sur la pupille. Par contre, si on effectue une restitution holographique optique où l'image est accompagnée d'une image conjuguée, il faut séparer l'image retenue aussi du réseau des images conjuguées ce qui revient en pratique à doubler la fréquence d'échantillonnage au moins selon $x$ ou $y$ par exemple ce qui astreint finalement à transcrire sur l'hologramme autant d'information mais sous forme réelle exclusivement. On peut montrer de plus que se superposent aux effets précédents de l'échantillonnage un lissage de l'image dû au fait que l'information retenue est en fait intégrée sur la surface non nulle des transducteurs utilisés (Annexe 2).

3. Les techniques. - L'application des concepts holographiques à l'imagerie acoustique a suscité à partir des années 67-68 un grand nombre de recherches expérimentales. Les rétines phonosensibles ainsi développées sont susceptibles d'être utilisées pour enregistrer un hologramme ou une image acoustique. On verra plus loin que dans certains cas la surface détectrice peut être associée à un dispositif optique assurant la conversion instantanée de l'information acoustique en information optique directement utilisable par les récepteurs optiques classiques, ce qui revient à assurer globalement le processus holographique sans avoir à fixer l'hologramme sur un film. Les travaux ainsi effectués sont déjà trop nombreux pour être cités tous ici. En l'absence de films phonosensibles de qualités comparables à celles des émulsions photographiques, le problème technologique essentiel demeure la réalisation de la rétine. Les recherches orientées vers le développement d'émulsions phonosensibles à l'aide de films thermosensibles de cristaux liquides n'ont pas abouti du fait de l'insuffisance des résultats obtenus en sensibilité et rapidité. Les surfaces réceptrices sont constituées le plus souvent à partir d'un réseau de transducteurs piézoélectriques ou d'un interface liquide-gaz ou liquide-solide ou solide-gaz dont les déformations encourues du fait de l'onde acoustique sont détectées optiquement. Il est difficile de présenter une sélection et une classification des méthodes proposées qui sont très diverses. On distinguera un peu arbitrairement :

- les techniques d'imagerie classiques utilisant des lentilles acoustiques associées à une rétine photosensible ;

- les techniques holographiques incluant certains dispositifs où l'holographie se réduit à un traitement du signal améliorant la sensibilité et la dynamique de réception ; 
- les techniques de focalisation à grande ouverture utilisant le balayage d'un transducteur focalisant réel ou d'une ouverture synthétique utilisant les informations cohérentes délivrées en parallèle ou séquentiellement par un grand nombre de transducteurs ponctuels répartis dans l'ouverture retenue et corrigées en phase par un traitement du signal adéquat.

3.1 Les TEChNIQUeS D'IMAgerie ClassiQue. 3.1.1 La caméra à ultrasons de Sokolov. - L'intérêt porté à l'imagerie ultrasonore s'est manifesté dès les premières utilisations des ultrasons au contrôle non destructif des matériaux. Toutes ces expériences d'imagerie sont effectuées en immersion dans l'eau. La première rétine phonosensible susceptible de fournir une image acoustique rapidement fut proposée par Sokolov en 1930 [7]. Depuis, et jusqu'à ces dernières années, diverses caméras ultrasonores ont été réalisées [8 à 13] s'inspirant de l'invention de Sokolov qui consiste à explorer par un faisceau électronique la surface interne d'une plaque piézoélectrique soumise au niveau de sa surface externe au champ acoustique à visualiser (Fig. 5). Cette plaque constitue la face avant
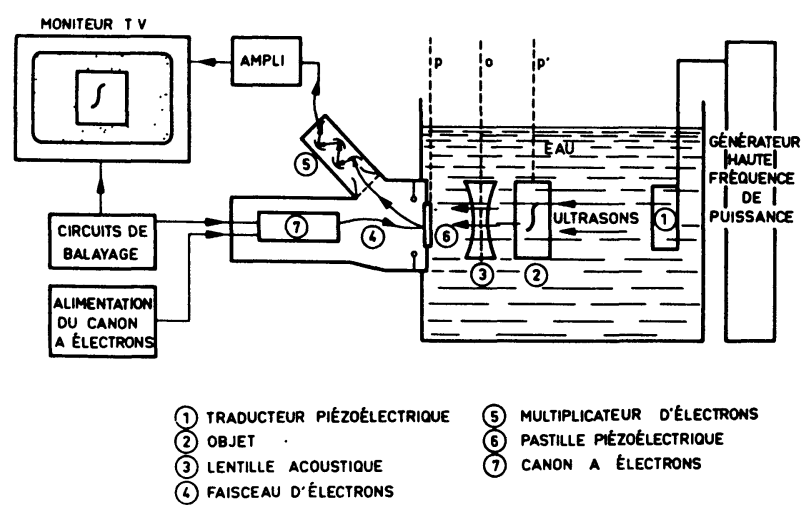

Fig. 5. - Caméra ultrasonore du type Sokolov (Marini [11]).

d'un tube cathodique analogue à celui d'un oscilloscope. La face externe de la plaque piézoélectrique qui peut être une lame de quartz ou de céramique en titanate de baryum est métallisée et reliée à la masse. Il apparaît, du fait du champ acoustique incident, un relief de potentiel sur la face interne dont l'effet est de moduler l'émission secondaire dont cette face isolante est responsable à l'impact du faisceau électronique d'exploration. Le courant secondaire est recueilli et amplifié par un photomultiplicateur qui délivre un signal $\mathrm{S}$ reflétant l'évolution du potentiel piézoélectrique imposé par le champ acoustique au niveau de la zone explorée électroniquement. Si on impose au faisceau électronique un balayage semblable à celui utilisé en télévision le signal $\mathrm{S}$ amplifié et détecté, appliqué au Wehnelt d'un oscilloscope synchronisé en balayage sur la caméra, permet de visualiser une image ordinaire d'intensité ultrasonore en temps réel. Une telle rétine, séduisante par sa rapidité, sa relativement grande sensibilité à des flux ultrasonores de l'ordre de $10^{-8}$ à $10^{-9} \mathrm{~W} / \mathrm{cm}^{2}$, présente cependant un assez grave inconvénient quant à la résolution du fait que la lame piézoélectrique, travaillant en général à la résonance pour sauvegarder la sensibilité, est surtout sensible aux ondes acoustiques à incidence normale et réduit de ce fait l'ouverture utile de la pupille utilisée pour focaliser l'image et en même temps la résolution. Par ailleurs la section utile d'exploration est limitée par la résistance mécanique de la lame soumise au vide sur sa face interne et à la pression atmosphérique à l'extérieur. Le nombre de points acoustiques séparables ou encore de franges acoustiques visualisables sur un diamètre reste très réduit et limite sensiblement la qualité des images. Diverses améliorations ont été proposées pour remédier aux deux défauts précédents mais n'ont pas cependant permis d'améliorer les résultats de manière très compétitive $[12,13]$ avec les techniques présentées plus loin.

3.1.2 Les lentilles acoustiques. - Pour des objets assez minces, il peut s'avérer suffisant de les irradier en les disposant près de la rétine en sorte qu'on obtient des images d'ombres dont la netteté se dégrade dès qu'on éloigne l'objet. Cependant, la résolution optimale ne peut en général être obtenue en imagerie classique qu'à l'aide d'une lentille acoustique dont la conception est tout à fait identique à celle des lentilles optiques, à la différence près qu'on dispose de milieux acoustiques de célérité aussi bien supérieure qu'inférieure à l'eau, c'est-à-dire d'indice inférieur ou supérieur à 1 . On peut ainsi réaliser des lentilles liquides convergentes à partir d'un liquide de célérité sonique inférieure à celle de l'eau, telles certaines huiles silicone, emprisonné entre deux diaphragmes qui affectent, du fait de la pression du liquide injecté, une déformation sensiblement parabolique, convenable pour des ouvertures angulaires pas trop grandes. Une autre méthode simple consiste à utiliser un bloc de plexiglass usiné comme une lentille optique divergente à faces sphériques mais qui, du fait de la célérité du plexiglass $(C \sim 2600 \mathrm{~m} / \mathrm{s})$ constitue encore une lentille convergente. Comme pour les lentilles optiques on observe des pertes par réflexion aux interfaces qui peuvent être réduites par un traitement de surface dans le cas des lentilles solides, analogue au bleuissement des objectifs photographiques, se traduisant en acoustique par une couche mince d'impédance intermédiaire entre le solide et l'eau. Un inconvénient purement acoustique réside dans l'apparition d'ondes transverses parasites, à l'intérieur d'une lentille solide, dont il faut aussi minimiser l'effet.

3.1.3 Une caméra ultrasonore fournissant des images d'intensité en temps réel de bonne qualité a été développée récemment par $\mathrm{Ph}$. Green [14]. Dans ce dispositif la rétine phonosensible est réduite à une barrette linéaire de 192 transducteurs grâce au subterfuge qui consiste à remplacer le déplacement mécanique apparemment nécessaire du réseau linéaire dans la direction perpendiculaire à son orientation par le déplacement re'atif équivalent de l'image acoustique, 


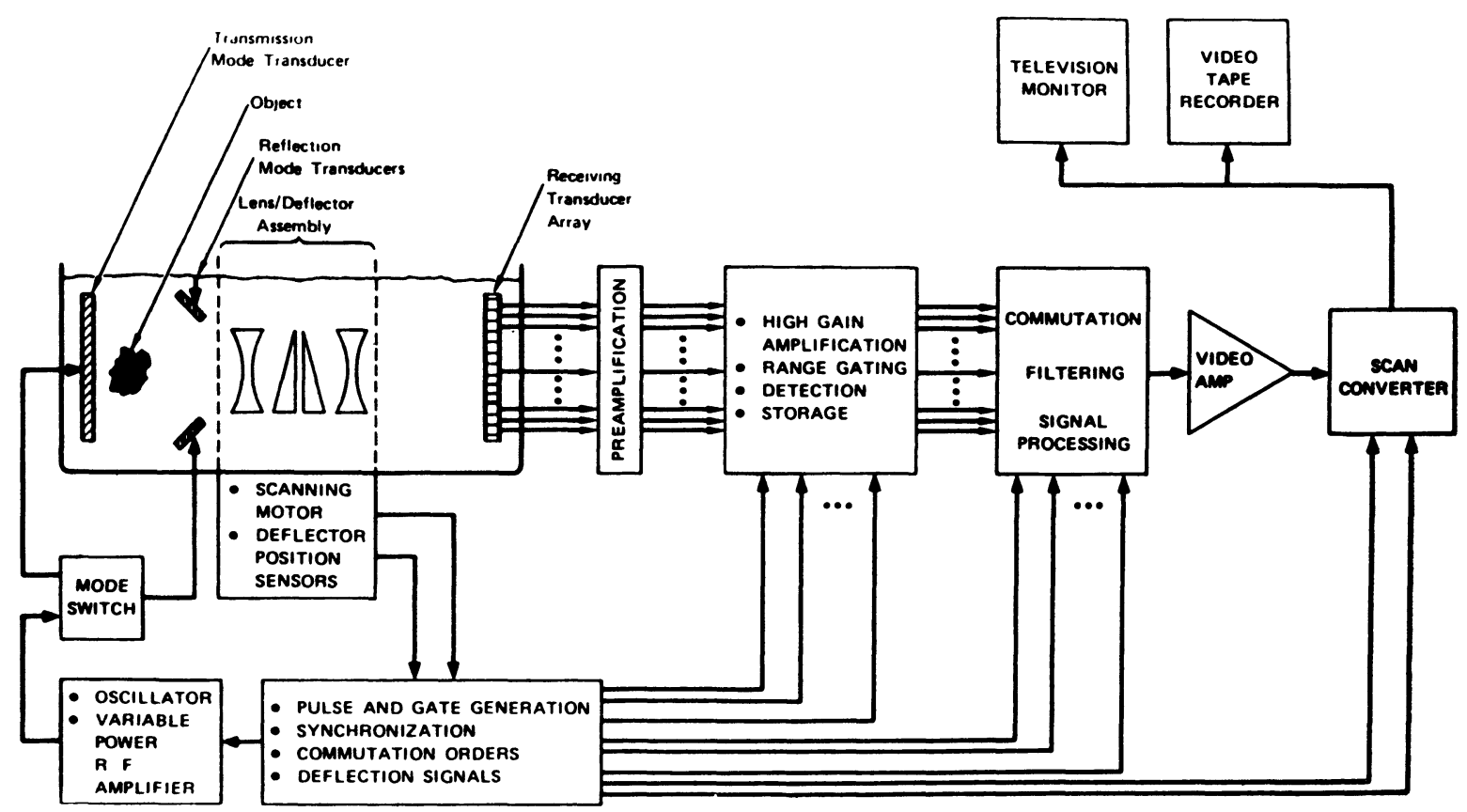

Fig. 6. - Caméra ultrasonore de Green [14].

obtenu à partir d'un système de prismes acoustiques contrarotatifs, comme l'indique la figure 6 , équivalent acoustique d'un dispositif utilisé classiquement en optique. La rotation mécanique des prismes assure l'obtention de 8 à 10 images par seconde sans créer de turbulence ou d'effets de convection dans l'eau capables d'altérer les résultats obtenus qui sont excellents pour des objets ou des organes excisés vus en transparence (Fig. 7). L'utilisation de 4 éléments réfringents et de 8 interfaces n'empêche pas de conserver une sensibilité

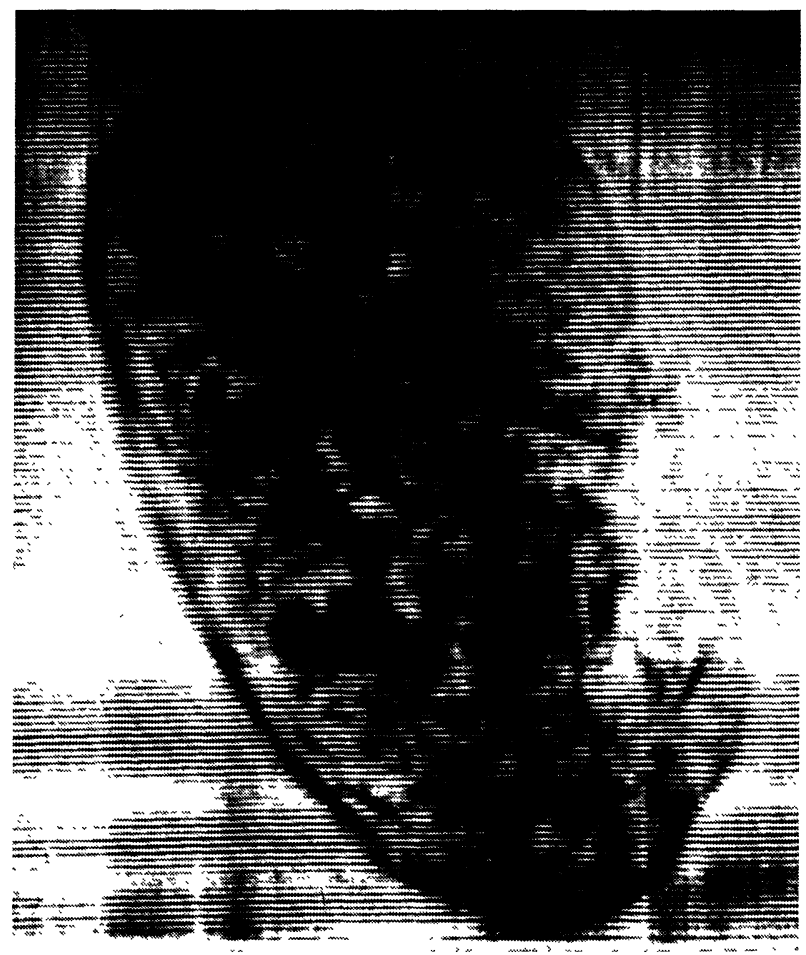

Fig. 7. - Image en transparence d'un rein excisé (Green). excellente $\left(10^{-9} \mathrm{~W} / \mathrm{cm}^{2}\right)$ compatible avec les applications médicales. Un film a été présenté récemment [15] donnant des images en temps réel et en transparence de l'abdomen.

3.2 TeChNiques holographiques. - 3.2.1 Enregistrement par balayage mécanique. - La méthode la plus simple pour enregistrer un hologramme acoustique consiste à explorer mécaniquement la pupille holographique à l'aide d'un récepteur omnidirectionnel quasi ponctuel. Comme on dispose alors directement du signal en amplitude et en phase il n'est pas nécessaire de faire appel à une onde de référence comme en optique et il est souvent plus avantageux d'effectuer l'équivalent électronique de l'interférence c'est-à-dire l'addition au signal reçu dans le plan des amplitudes complexes d'un signal de référence avant la détection. Un tel procédé de détection en phase connu sous le nom de détection synchrone est connu pour améliorer notablement le rapport signal sur bruit du fait qu'il est équivalent à un filtre passe bande, adapté à la fréquence de travail. En général, pour une raison de simplicité, le signal de référence utilisé est en synchronisme de phase avec le signal responsable de l'illumination acoustique et s'avère au point de vue spatial de phase constante c'est-à-dire équivalent à une onde de référence d'incidence normale ce qui exige alors une illumination oblique de l'objet pour obtenir la séparation des images à la restitution. Naturellement un tel traitement électronique du signal est très souple et, malgré la lenteur inhérente au procédé, il faut en général plusieurs minutes pour un enregistrement. De nombreuses expériences à caractère fondamental furent effectuées ainsi pour tester l'holographie acoustique. Les figures 8 montrent les résultats ainsi obtenus 

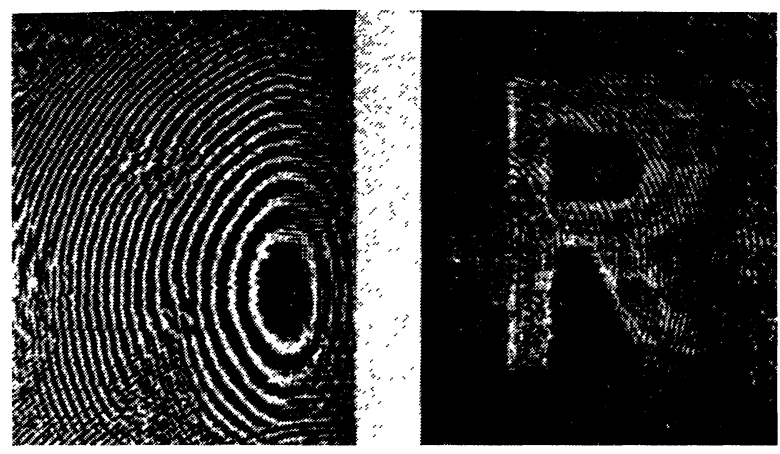

Fig. 8. - Hologramme et image restitués de la lettre $R$ (Metherell).

par Metherell [16] avec des ultrasons dans l'air à $20 \mathrm{kHz}$ $(\lambda \sim 1,5 \mathrm{~cm})$ utilisés pour observer acoustiquement des lettres de polystyrène de la taille du mètre. On notera sur la figure 9 les précautions prises pour éliminer lors de la restitution optique l'onde non perturbée et l'onde conjuguée à l'aide d'un filtre spatial disposé au foyer de la lentille $L_{2}$.

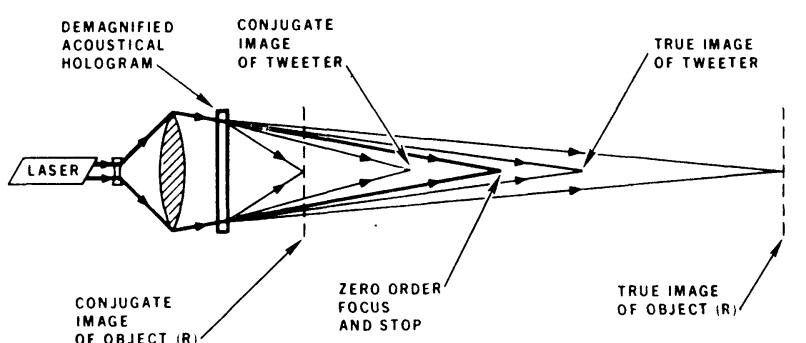

FIG. 9. - Dispositif optique de restitution holographie (Metherell)

3.2.2 La méthode du relief de surface. - Les liquides mettent particulièrement bien en évidence les effets non linéaires des ultrasons et peuvent être notablement mis en mouvement (vent acoustique) ou déformés en surface par la pression de radiation. C'est ce dernier phénomène qui a permis le développement d'une rétine phonosensible remarquable.

Une onde acoustique se propageant dans un liquide se réfléchit à la surface de celui-ci en créant d'une part, une déformation faible à la fréquence acoustique selon les lois bien connues de la réflexion d'autre part, une déformation statique créée par la pression de radiation $p_{\mathrm{R}}$ qu'on peut considérer comme un effet non linéaire de fréquence nulle [17]. La très grande déformabilité de la surface est seulement limitée par la gravitation et la tension superficielle $\gamma$ du liquide en sorte que la lévitation $\zeta(x, y)$ est dictée par l'équation

$$
\rho_{0} g \zeta-\gamma \nabla^{2} \zeta=p_{\mathrm{R}}(x, y)=\frac{1}{2} \rho_{0} \overline{\left(\frac{\partial \varphi}{\partial z}\right)^{2}},
$$

la pression de radiation étant liée à la moyenne temporelle du carré de la vitesse acoustique normale à l'interface.

Si dans ces conditions on relie l'information acoustique à la vitesse normale $V_{z}=\partial \varphi / \partial z$ induite par l'onde incidente, la réponse de l'interface s'exprime simplement dans l'espace des fréquences spatiales:

$$
Z(\mathbf{f})=\left[g\left(1+l^{2} f^{2}\right)\right]^{-1} A A^{*}=H A A^{*},
$$

où $Z$ et $A$ sont les transformées de Fourier de $\zeta$ et $U$ et $l=2 \pi\left(\gamma / \rho_{0} g\right)^{1 / 2}$ une longueur caractéristique qui est de l'ordre de $2 \mathrm{~mm}$ pour l'interface eau-air. Dans l'espace ordinaire cette relation s'écrit

$$
\zeta=U U^{*} * h, \quad h=T F^{-1}(H),
$$

où $h(x, y)$ représente la réponse impulsionnelle (au sens spatial) de l'interface. Une telle rétine opère une détection quadratique comme l'émulsion photographique et exige donc pour son emploi en holographie l'utilisation d'une onde de référence comme en optique (fig. 10).

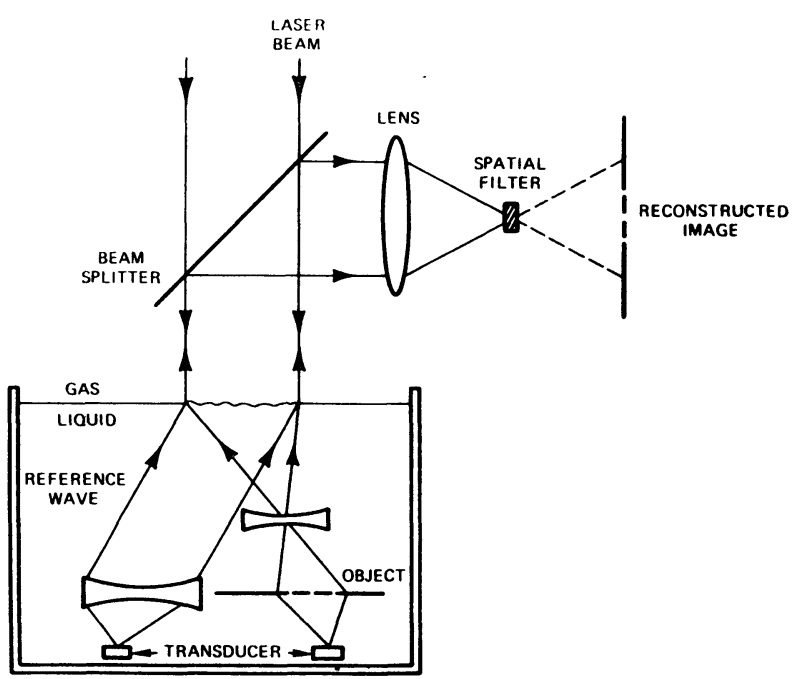

Fig. 10. - Holographie par relief de surface.

On constate au niveau de la relation (45) que le terme $H(\mathbf{f})$ opère comme un filtre passe bas et atténue les fréquences spatiales élevées qui sont en général les plus intéressantes. C'est pourquoi dans les montages pratiques, on interpose au niveau de l'interface une cuve de faible profondeur, dont le fond est constitué d'un film plastique transparent à la radiation ultrasonore, remplie d'un liquide à faible tension superficielle permettant de réduire la longueur $l$. L'onde plane de référence est inclinée relativement à l'onde plane illuminant l'objet de manière à obtenir un système de franges linéaires en l'absence d'objet dont la perturbation par ce dernier constitue l'information holographique. La déformation $h$ induite sur l'interface est alors détectée optiquement pour fournir un hologramme traité classiquement ou, mieux encore, permet d'obtenir directement par la réflexion d'un faisceau de lumière cohérente incident une onde diffractée en plusieurs ordres qu'on peut évaluer par un calcul analogue à celui classique de la diffraction de Raman Nath de la lumière par les ultrasons. L'ordre 1 (ou - 1) sélectionné par un écran disposé dans le plan focal de la lentille optique utilisée est identique à l'onde qu'on peut restituer par un holo- 
gramme en transparence sans modification d'échelle et permet d'observer une image optique de l'objet en temps réel. En pratique, l'observation d'une onde diffractée de puissance faible $\left(10^{-3} \mathrm{~W} / \mathrm{cm}^{2}\right)$ exige l'utilisation d'une onde de référence notablement plus puissante $\left(10^{-1}\right.$ à $\left.1 \mathrm{~W} / \mathrm{cm}^{2}\right)$ pour créer une déformation convenable optiquement de l'ordre de $1000 \AA$. Le montage holographique ainsi décrit est alors très sévèrement touché par les aberrations induites par la non-uniformité de l'onde de référence, de même que par les oscillations parasites de la cuve induites par les vibrations du sol qui correspondent les unes et les autres à des fréquences spatiales faibles qu'on peut en principe éliminer par filtrage optique sauf, et c'est en général le cas, si les déformations associées sont supérieures à la longueur d'onde optique utilisée [19]. C'est pourquoi les meilleurs résultats ont été d'assez loin obtenus par une méthode développée par Smith et Brenden [20] consistant à focaliser une image acoustique de l'objet à l'aide d'une lentille acoustique au niveau de l'interface. Le traitement optique de restitution holographique se réduit alors à fournir une image optique ordinaire d'intensité à partir de l'image acoustique de phase. Les aberrations acoustiques de basse fréquence spatiale sont alors facilement éliminées par un filtrage optique dans le plan focal de la lentille optique et n'ont aucun effet sur les fréquences spatiales élevées. Les premières belles images acoustiques furent ainsi obtenues assez rapidement (Fig. 11) grâce à des améliorations diverses consistant notamment à utiliser une illumination acoustique modulée en trains d'ondes de quelques dizaines d'oscillations interdisant l'apparition des aberrations trop fortes et une illumination optique également pulsée en synchronisme convenable.

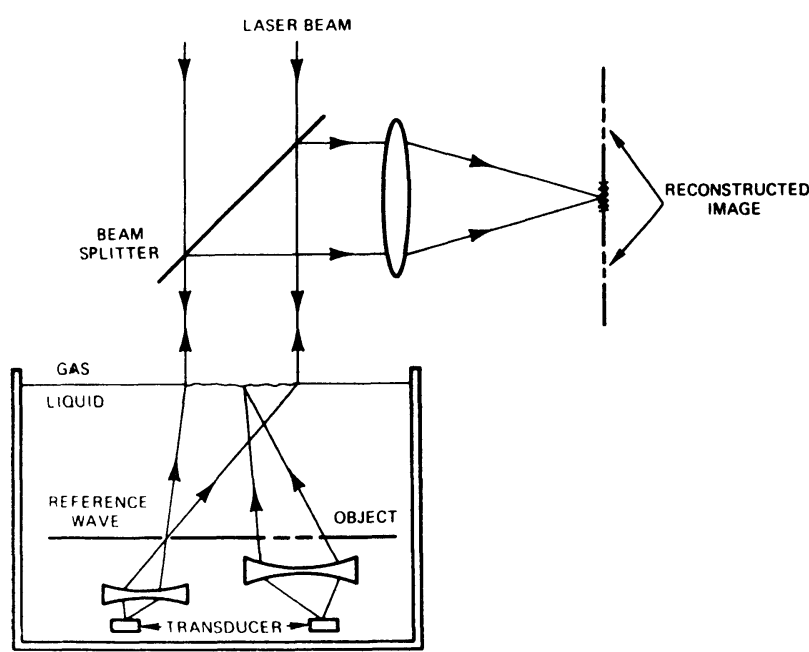

Fig. 11. - Montage holographique de Breden.

3.2.3 Détection optique en amplitude et phase de la déformation acoustique d'un interface. - L'utilisation d'un interface comme surface continue de réception présente un grand intérêt en ce qui concerne l'ouverture et la résolution du montage et permet en principe d'enregistrer un très grand nombre de franges acous- tiques. C'est pourquoi, malgré l'amplitude extrêmement faible, de l'ordre de l'angström, de la déformation subie à la fréquence acoustique par l'interface, plusieurs techniques ont été proposées pour mesurer cette dernière :

- L'interférométrie holographique optique imaginée par Metherell [21]. La figure $12 a$ montre le schéma de l'opération qui consiste à enregistrer sur une même plaque photosensible un double hologramme optique de l'interface en des temps séparés d'une demi-période acoustique et avec une référence optique décalée en quadrature de phase d'une exposition à l'autre. La figure $12 b$ montre comment, dans des

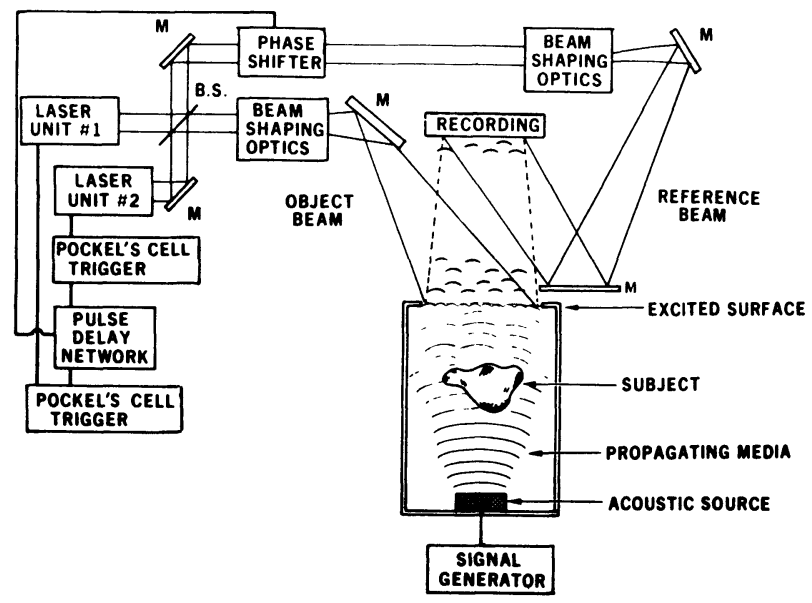

Interférométrie Holographique

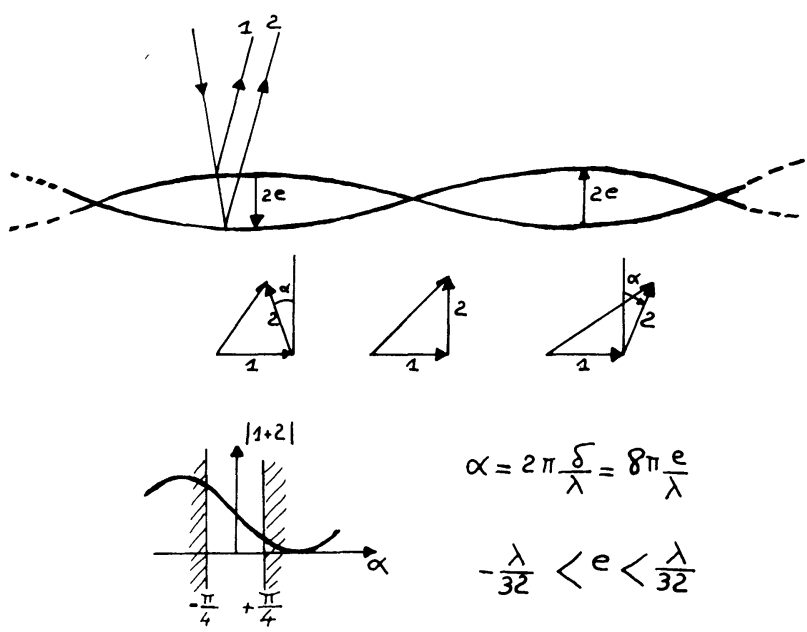

Fig. 12. - a) Holographie ultrasonore par interférométrie holographique optique (Metherell). b) La variation de phase encourue de 1 à 2 est convertie en variation d'amplitude comme en microscopie de phase.

conditions analogues à celles de la microscopie de phase, un objet de phase se traduit par une image d'intensité. L'interface liquide-gaz, le plus favorable pour la sensibilité, reste utilisé mais avec l'adjonction d'un film plastique métallisé très fin tendu en surface qui, sans perturber notablement la déformation étudiée, permet une bonne réflexion optique et surtout inhibe les effets quasi statiques non linéaires utilisés 
par la technique précédente. La figure $12 b$ permet de comprendre que la gamme de déformation mesurable par ce procédé est limitée vers le haut à $\lambda_{0} / 32$ où $\lambda_{0}$ est la longueur d'onde optique, mais c'est la limitation inférieure correspondant au contraste minimum enregistrable qui est surtout gênante. Elle correspond sensiblement [21] à un déplacement associé à $10^{-3} \mathrm{~W} / \mathrm{cm}^{2}$ à $1 \mathrm{MHz}$ soit à peu près à $5 \AA \AA$. On notera que la figure de franges est obtenue par un effet stroboscopique, la référence étant fixée cette fois par l'instant de déclenchement de la prise de vue. Une telle référence temporelle est, elle, aussi équiphase spatialement et équivalente à une onde de référence à incidence normale.

— L'interférométrie optique à excursion séquentielle.

Un montage proposé récemment [22] consiste à utiliser un film métallisé tendu à l'intérieur de la cuve. Il n'y a plus d'interface à proprement parler mais une surface plane matérialisée par le film laquelle subit la déformation normale acoustique sans réfléchir ni perturber beaucoup cette dernière. Un dispositif de balayage optique à miroirs croisés permet alors d'explorer cette surface par un pinceau laser à incidence normale qui est modulé en phase par le déplacement acoustique et un interféromètre de Michelson associé permet de convertir l'information de phase en information d'amplitude lue par une photodiode. Malgré l'inconvénient présenté par l'échantillonnage séquentiel de la rétine, les performances annoncées sont bonnes du fait du traitement électronique du signal.

- La caméra ultrasonore de Korpel Desmares [23]. L'exploration séquentielle d'un interface est là aussi effectuée par un pinceau laser (Fig. 13) mais l'information est fournie par les modifications apportées à la réflexion liée à la courbure subie par l'interface et reçue à partir d'un photodétecteur à couteau. La sensibilité élevée de ce procédé autorise l'emploi d'un interface

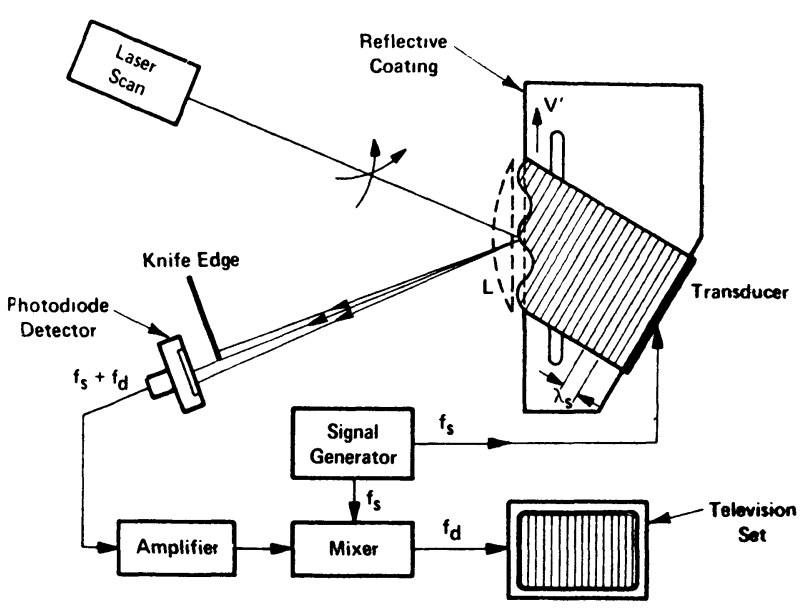

Fig. 13. - Caméra ultrasonore de Korpel Desmares [23].

liquide-solide et a permis de développer une technique tout à fait remarquable de microscopie acoustique à des fréquences de 100 à $200 \mathrm{MHz}$ permettant de visualiser des échantillons biologiques minces avec une résolution de l'ordre de $30 \mu$. Dans la mesure où l'échantillon est plaqué contre l'interface, son image apparaît pratiquement correcte sur ce dernier et bien que l'information reçue soit susceptible de fournir un hologramme traité par une restitution optique classique il est plus avantageux de faire apparaitre directement par un traitement électronique adéquat cette image d'ombres portées en temps réel sur un moniteur de télévision. Cette technique a reçu un développement technologique très poussé [24] permettant la superposition d'une image acoustique rouge, et d'une image optique bleue obtenue par détection de la transmission du faisceau laser d'exploration, sur l'écran d'un moniteur de télévision couleur autorisant la comparaison et l'utilisation des informations délivrées par les deux rayonnements.

- Gabor a proposé aussi l'utilisation d'un film

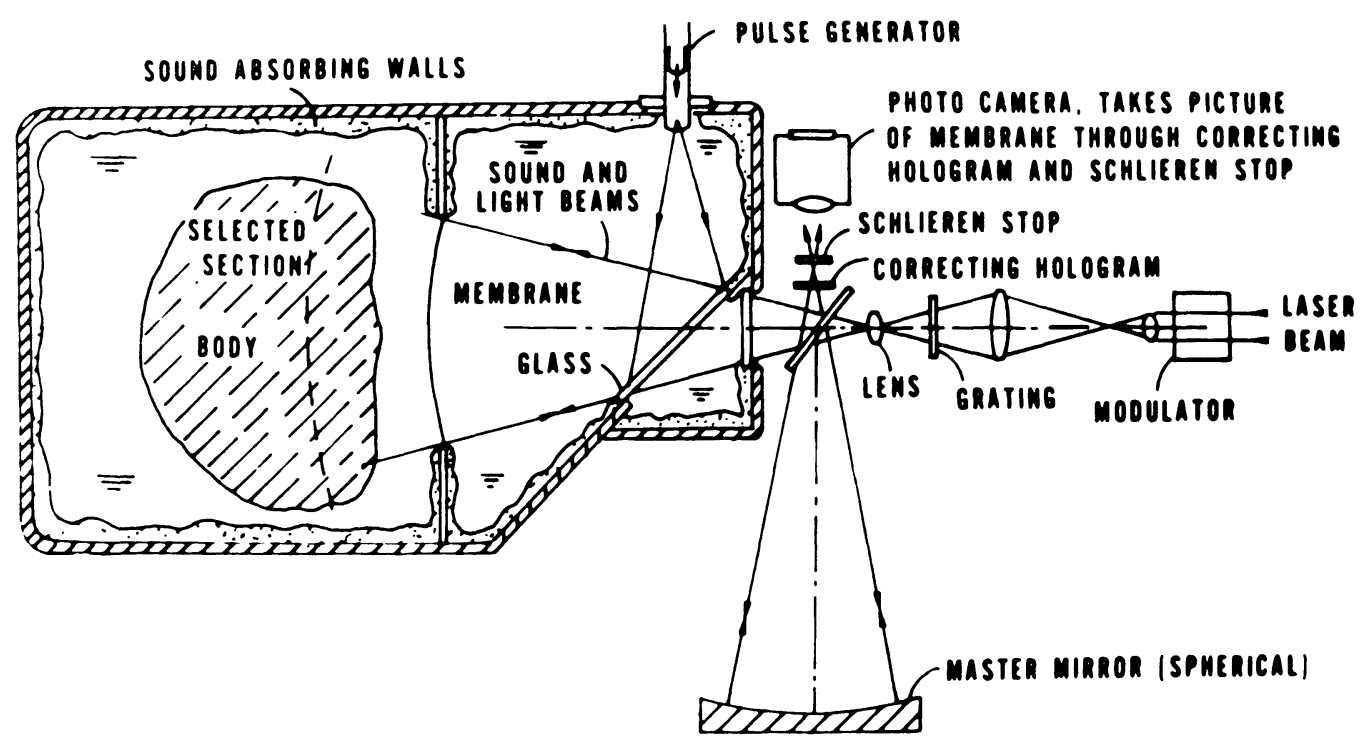

Fig. 14. - Caméra ultrasonore de Gabor [25]. 
plastique métallisé (Fig. 14) [25] associée à une illumination acoustique se réduisant à une impulsion unique. Un point diffractant de l'objet renvoie alors une onde sphérique se traduisant à un instant donné par une déformation en anneau sur le film métallisé détectable optiquement à l'aide d'un flash et d'un système optique fournissant l'image du film sur une plaque photographique. Une série de flashes déclenchés à des intervalles de temps réguliers $T$ permet alors d'obtenir sur la plaque d'enregistrement la superposition des anneaux selon la configuration classique de Fresnel caractéristique de l'hologramme d'un point. De la même façon un objet quelconque permet d'obtenir un hologramme équivalent à celui que fournirait un rayonnement ultrasonore de période $T$.

3.2.4 Réseaux matriciels de transducteurs. - Aussi séduisant que soit l'emploi d'un interface comme rétine phonosensible il semble difficile d'atteindre de cette façon les performances en sensibilité et dynamique de réception offertes par un simple transducteur piézoélectrique. C'est pourquoi plusieurs équipes de recherches s'attachent à développer une technologie de réseaux matriciels de transducteurs piézoélectriques. La difficulté naît de ce que la résolution souhaitée exige des rétines de plusieurs milliers ou plusieurs dizaines de milliers de transducteurs et la complexité ne provient pas tant de la réalisation de la mosaïque piézoélectrique que de celle des circuits électroniques et des connexions associés. Pour limiter les fils et les composants électroniques, une solution consiste en une commutation adressable en $x$ et $y$ de chaque transducteur à un circuit de réception unique (méthode séquentielle) ou adressable en $y$ de chacun des $n$ transducteurs de la ligne correspondante à $n$ circuits de réception montés en parallèle (méthode semi-parallèle), ce dernier cas s'imposant pratiquement si on veut sauvegarder le temps réel. On peut aussi citer quelques réalisations effectuées avec des composants discrets de rétines à quelques milliers de points [26] où la commutation se réduit pratiquement à une diode et une résistance par transducteur. Cependant l'avenir est à cet égard aux réalisations électroniques intégrées. Plusieurs études sont en cours orientées soit vers la commutation directe par transistors à effet de champ intégrés directement sur la face arrière d'un cristal de niobate de lithium utilisé comme élément piézoélectrique, commutation adaptée à l'émission ou à la réception c'est-àdire à des signaux forts (100 à $200 \mathrm{~V})$ et faibles [27], soit vers la réalisation intégrée sur un cristal de même nature d'un préamplificateur et d'une commutation pour un fonctionnement exclusivement en réception.

En attendant que ces recherches, qui posent des problèmes technologiques très ardus, aboutissent, une solution souple et peu onéreuse consiste en l'emploi en commutation semi-parallèle d'un réseau matriciel de transducteurs électrostatiques [28, 29]. De tels transducteurs, version ultrasonore du classique microphone à condensateur, peuvent être réalisés à partir d'un simple sandwich où un film diélectrique adéquat est enserré par un circuit imprimé rigide face arrière et un circuit imprimé souple (face avant). Une matrice de $256 \times 256$ points (Fig. 15) a ainsi été réalisée à partir

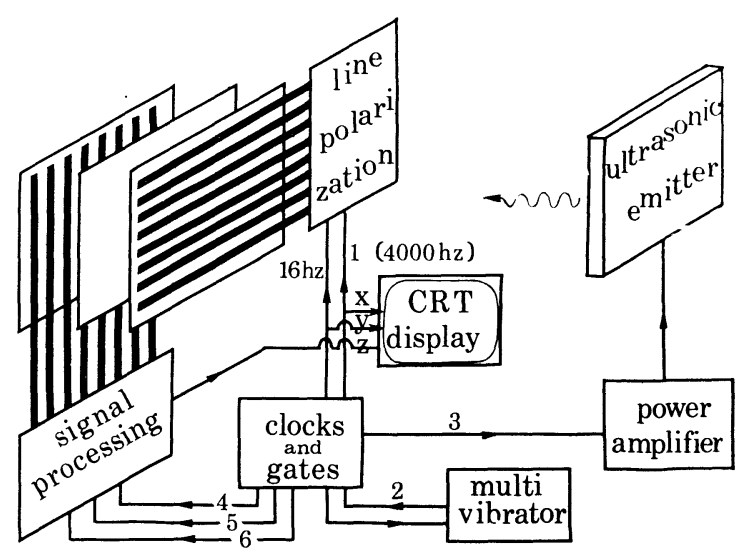

FIG. 15. - Rétine phonosensible électrostatique [29].

de deux circuits photogravés selon 256 électrodes linéaires parallèles disposées orthogonalement. La commutation semi-parallèle consiste à polariser séquentiellement les lignes. Les transducteurs associés à la ligne polarisée, seuls à fonctionner, transmettent alors leur information via les colonnes à 256 circuits de réception montés en parallèle. Une récurrence de polarisation de ligne de $4000 \mathrm{~Hz}$ autorise pendant les $250 \mu$ s alloués à chaque ligne un temps de réception de $100 \mu$ s et le multiplexage des valeurs obtenues par détection synchrone et intégration et mémorisées sur capacités en $80 \mu$ s à la cadence de $2,5 \mathrm{MHz}$ environ. Les $(256)^{2}$ points de la matrice sont ainsi explorés à la cadence d'environ 16 images par seconde ce qui préserve le temps réel pour l'observation directe sur le moniteur synchronisé en $X$ et $Y$ sur les récurrences de ligne et d'image et excité en $Z$ par le signal vidéo ainsi fourni. Les détections synchrones effectuées à partir d'un signal de référence en synchronisme avec le signal d'émission fournissent une information holographique correspondant à une onde de référence à incidence normale et l'onde plane acoustique utilisée pour éclairer l'objet doit être oblique pour séparer les images directe et conjuguée, ce qui donne un système de franges linéaires en l'absence d'objet interposé. Les figures $16 a$ et $16 b$ représentent un hologramme obtenu en interposant des lettres de liège opaques à la radiation ultrasonore de $2 \mathrm{MHz}$ utilisée et l'image restituée optiquement à partir d'un laser HeNe. Naturellement on peut aussi utiliser cette rétine en imagerie ordinaire mais au lieu de supprimer la détection synchrone, avantageuse dans la mesure où elle accroît la sensibilité de réception, on effectue un traitement du signal consistant à substituer au signal holographique $\mathrm{A} \cos \varphi$ sa valeur absolue légèrement lissée laquelle évolue sensiblement comme $|\mathrm{A}|$. Le nombre de franges initiales qui peut être de l'ordre 60 à 80 permet dans ces conditions l'obtention d'images d'assez bonne qualité (Fig. 17, 18). 

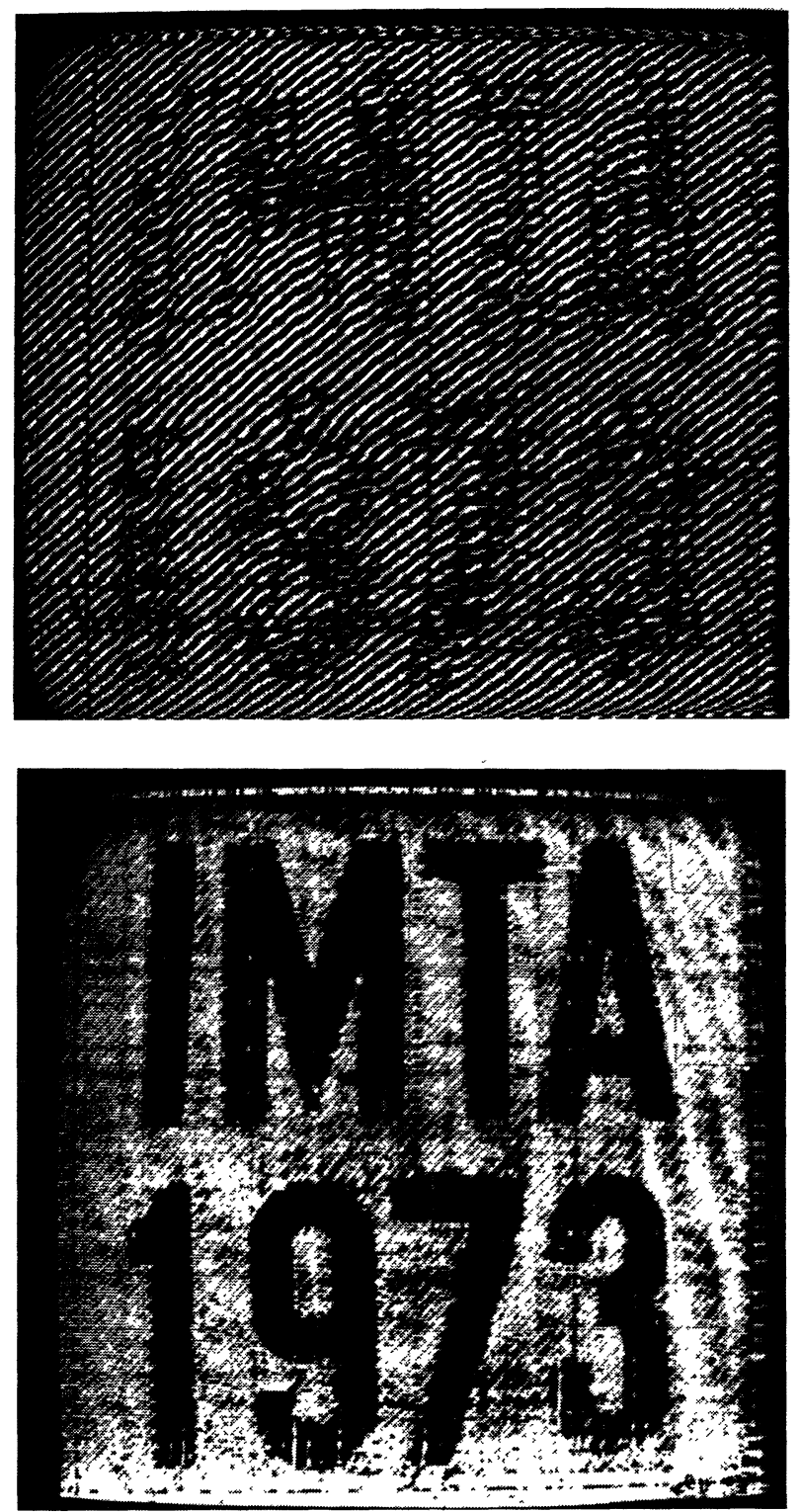

FIG. 16. - Hologramme (a) et restitution optique (b) (2 MHz) de lettres de lièges.

3.2.5 Imagerie par diffraction acousto-optique de Bragg. - Cette technique développée par Korpel [30] est nettement distincte des précédentes en ce que la reconstruction d'une onde optique restituant l'information ultrasonore procède d'un hologramme non pas superficiel mais réparti dans le volume de l'interaction acousto-optique un peu comme en holographie optique effectuée avec des émulsions photographiques épaisses. On sait que l'interaction acousto-optique faible revient à faire apparaître localement deux termes de source lumineuse dont les phases sont respectivement somme et différence des phases acoustique et optique des ondes incidentes. Lorsqu'il s'agit de l'interaction d'ondes planes dans un volume assez grand ces termes sources ne peuvent fournir de contribution constructive que si les ondes incidentes sont orientées de manière à ce que le vecteur d'onde diffractée puisse fermer le triangle initié par les vecteurs optique et acoustique incidents, comme l'indique la figure 19. Le déplacement

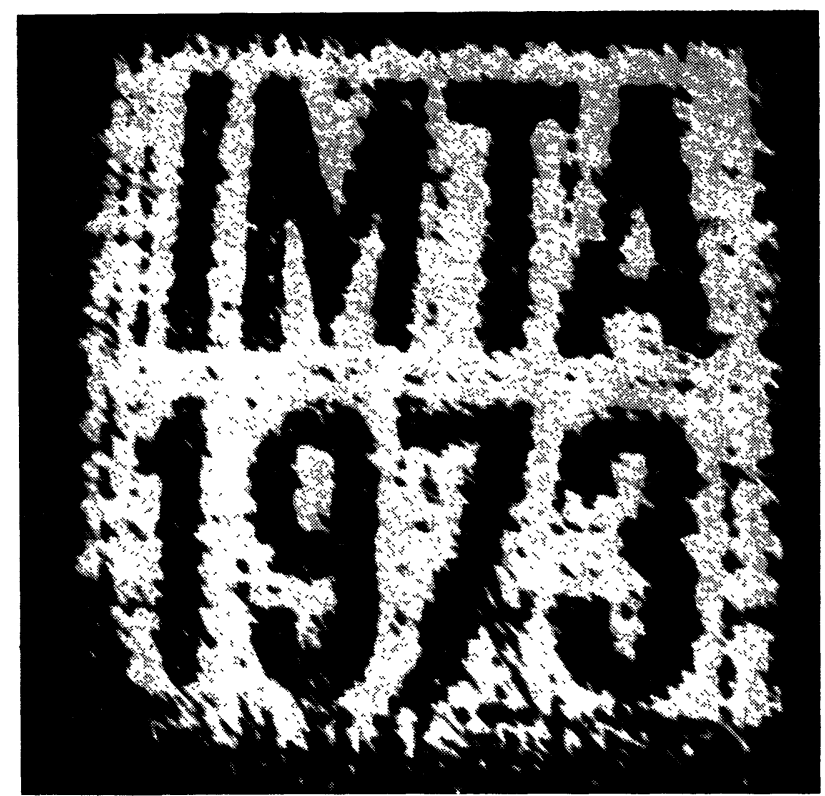

FIG. 17. - Image d'ombres (2 MHz).

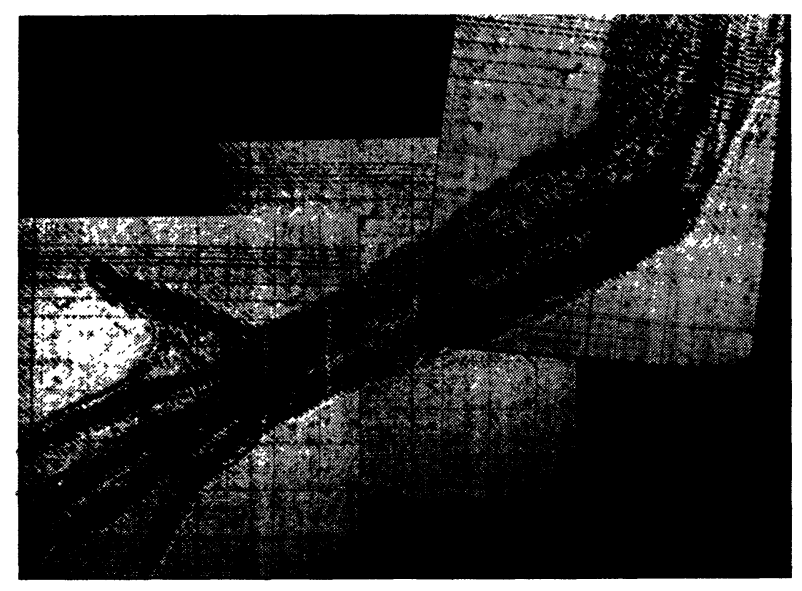

FIG. 18. - Image d'ombres (2 MHz).

de fréquence optique égal à la fréquence acoustique étant négligeable, ce triangle est sensiblement isocèle et son angle au sommet égal à $2 \varphi_{\mathrm{B}}$ (où $\varphi_{\mathrm{B}}$, l'angle de Bragg, est égal à $\lambda_{0} / 2 \lambda_{\mathrm{A}}$ ), c'est-à-dire au rapport des longueurs d'onde optique et acoustique, de l'ordre de $10^{-2}$ dans les cas pratiques. Ce phénomène n'est nettement mis en évidence que si la longueur du domaine d'interaction dans le sens parallèle aux plans d'onde acoustique est suffisante. La quasi-orthogonalité des ondes autorise l'utilisation d'un simple faisceau de lumière monochromatique. L'utilisation d'une lumière cohérente permet d'observer des diffractions plus complexes. C'est ainsi qu'un raisonnement géométrique élémentaire montre selon la figure 19 que l'interaction des deux ondes cyclindriques acoustique et optique donne lieu à deux ondes cylindriques optiques. Dans le plan de la figure, l'image I représentée correspond à la source ligne acoustique $\mathrm{A}$ dans la correspondance de similitude de centre $\mathrm{O}$ (ligne optique) d'angle $\left(\pi / 2-\varphi_{B}\right)$ et de rapport $\lambda_{0} / \lambda_{A}$ et de même l'image I' non représentée serait associée à la similitude 


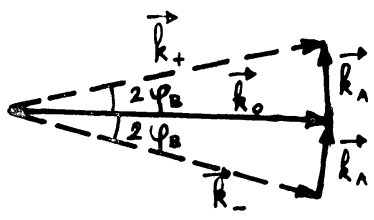

$$
\begin{aligned}
& \vec{k}_{ \pm}=\vec{k}_{0} \pm \vec{k}_{A} \\
& \varphi=\varphi_{0} \pm \varphi_{A}
\end{aligned}
$$

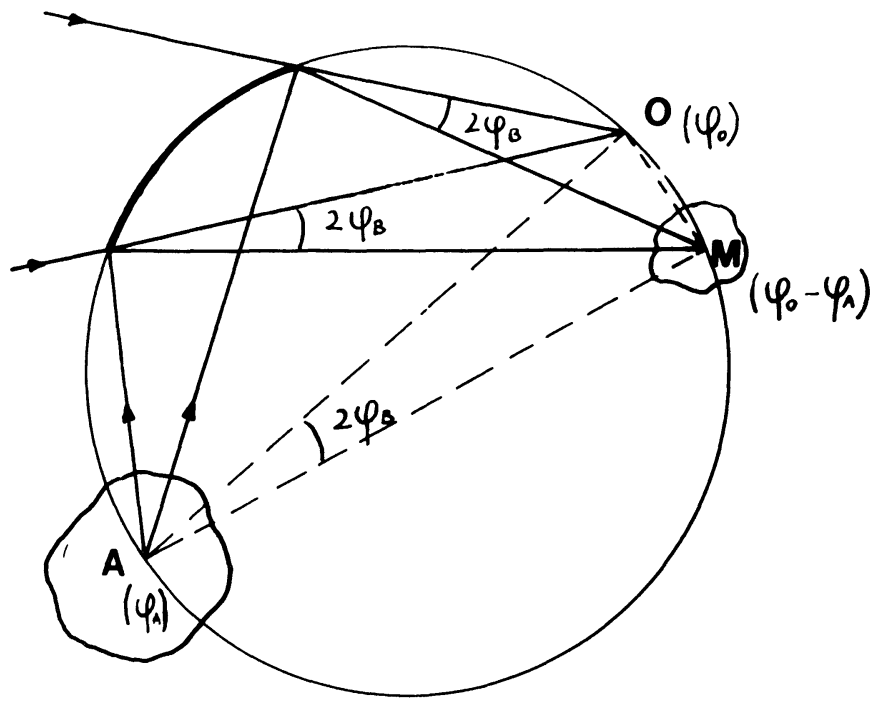

FIG. 19. - Stigmatisme acousto-optique par interaction de Bragg.

d'angle opposé. L'approximation admise par Korpel et confirmée par l'expérience consiste à admettre que l'interaction de l'onde optique cylindrique et d'une source ponctuelle A se traduit, du fait que l'interaction s'effectue essentiellement dans le plan issu de A normal à la ligne optique, selon deux ondes donnant deux images quasi ponctuelles I et $\mathrm{I}^{\prime}$ associées à A comme il vient d'être dit. Dans ces conditions, le phénomène substitue à l'objet acoustique (A) une image optique (I) ou l'image conjuguée $\left(\mathrm{I}^{\prime}\right)$ déduites de l'objet par une correspondance se réduisant à l'identité pour la coordonnée dans la direction de la ligne source et à une similitude réduisant les dimensions dans le rapport $\lambda_{0} / \lambda_{\mathrm{A}}$ dans le plan perpendiculaire. D'où une sévère déformation affine de l'image qui doit être compensée par un système anamorphoseur (Fig. 20). Cette tech-

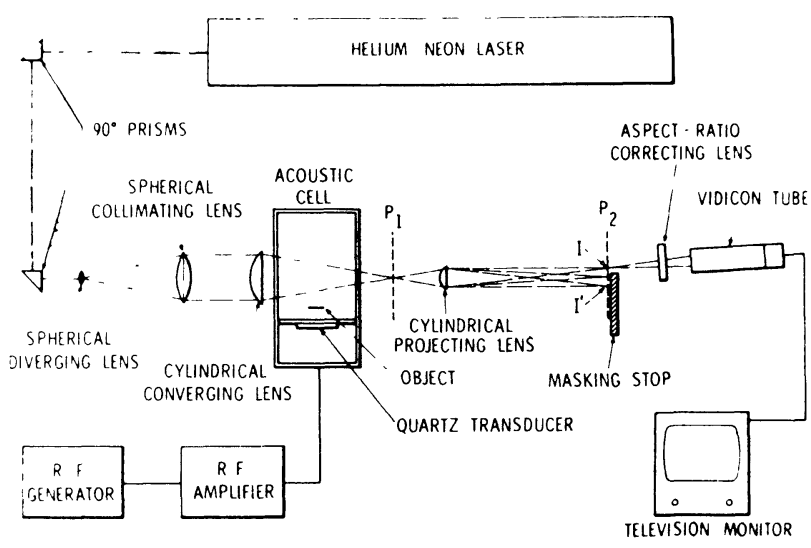

Fig. 20. - Voir Landry J., Powers J., Wade G., Ultrasonic Imaging of Internal structure by Bragg Diffractions, Appl. Phys. Lett. 15 (1969) 186 [31] et LANDRY J., SMITH R., WADE G., Optical detection in Bragg Imaging, Acoust. Holog. 3 (1972) 47-69. [32].

nique s'avère intéressante par sa relative simplicité et sa bonne adaptation aux fréquences ultrasonores élevées (10-30 MHz) pour le contrôle non destructif de petites pièces métalliques (Fig. 21), [31], [32].

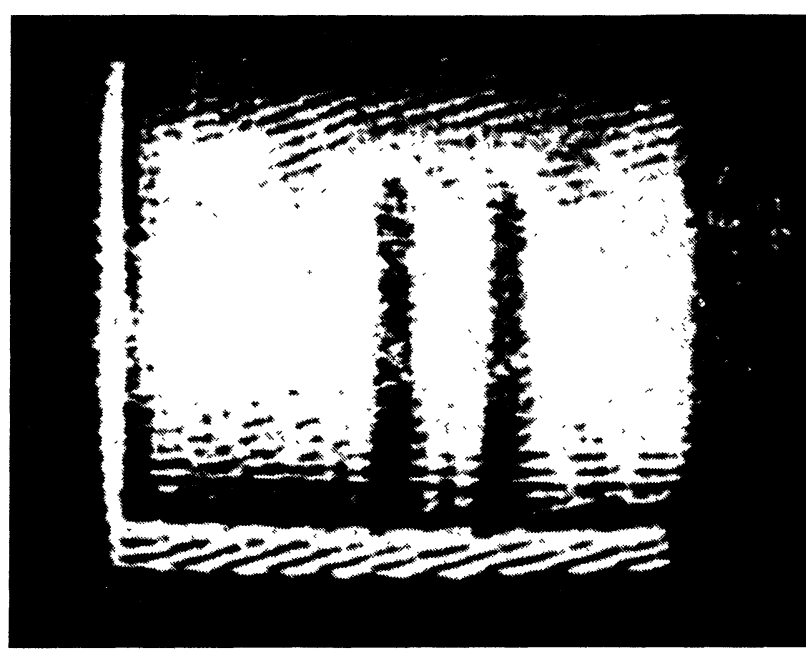

Fig. 21. - Pièce de duralumin percée de deux trous de $0,5 \mathrm{~mm}$ de diamètre visualisé à la fréquence ultrasonore de $25 \mathrm{MHz}$ ([31], [32]).

3.3 IMAGERIE A OUVERTURE MOBILE RÉELLE OU SYNTHÉTIQUE. - Les succès obtenus par les méthodes échographiques reposant essentiellement sur l'illumination sélective de la cible ont encouragé le développement dans ces dernières années de techniques d'imagerie en transparence ou d'échographie dite C (pour la distinguer de l'échographie B donnant des images en coupe) consistant à balayer mécaniquement ou électroniquement un transducteur focalisant à grande ouverture pour explorer une image en élévation de l'objet.

3.3.1 Balayage mécanique. Microscopie acoustique. - Un transducteur focalisant utilisé en émetteur récepteur (échographie $\mathrm{C}$ ) ou en récepteur exclusivement (imagerie par transparence) est déplacé selon un nombre adéquat de lignes (quelques centaines) pour fournir une image convenable et le signal qu'il transmet traité éventuellement dans le temps (porte électronique) et en amplitude puis restitué à l'aide du 
wehnelt d'un moniteur balayé en synchronisme ou à partir d'une lampe fixée sur l'équipage mécanique et photographiée par un appareil ouvert en pose pendant la durée, longue malheureusement, de l'opération. Cette technique simple a permis d'obtenir de remarquables images (Fig. 22) [34] et suscite toujours l'intérêt

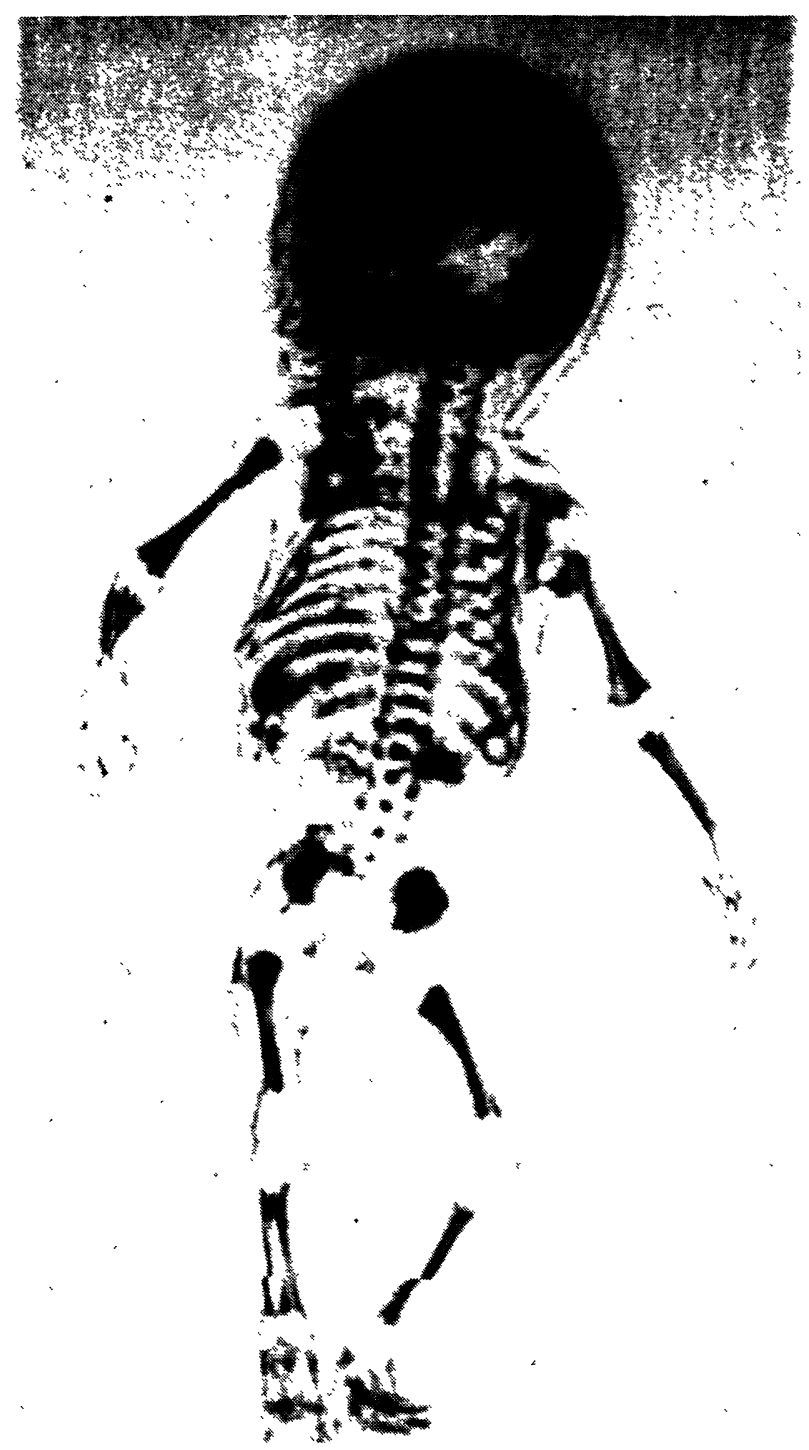

FIG. 22. - Radiographie d'un fœtus (Green et al. [34]).

pour l'étude systématique du comportement diffractant d'organes excisés. La lenteur inhérente au balayage mécanique devient beaucoup moins prohibitive dans certains cas où l'objet examiné est de petites dimensions, tels que l'examen échographique de l'œil [35] ou la microscopie acoustique. Dans ce dernier cas, c'est la coupe microscopique elle-même, déposée sur un film de Mylar très fin, qui peut être déplacée rapidement relativement au transducteur fixe, à une récurrence de ligne de l'ordre de $100 \mathrm{~Hz}$, ce qui permet la prise de vue en des temps de l'ordre de la seconde. C'est la méthode finalement retenue par l'équipe de Stanford orientée vers la microscopie acoustique depuis de nombreuses années [36, 37]. Les résultats récemment présentés [38] correspondent à une résolution de l'ordre du micron obtenue à des fréquences de 600 à $900 \mathrm{MHz}$. La résolution excellente, proche de l'optimum autorisé par la longueur d'onde, est acquise par l'emploi d'un transducteur focalisant à très grande ouverture $(f / 0,6)$ de faible aberration du fait que le dioptre sphérique focalisant utilisé (saphire-eau) correspond à un rapport d'indice de l'ordre de 8 et focalise très près du centre de courbure (Fig. 23). Ces résultats semblent d'ores et déjà placer la microscopie acoustique sur le terrain de l'utilisation par les médecins et les biologistes.
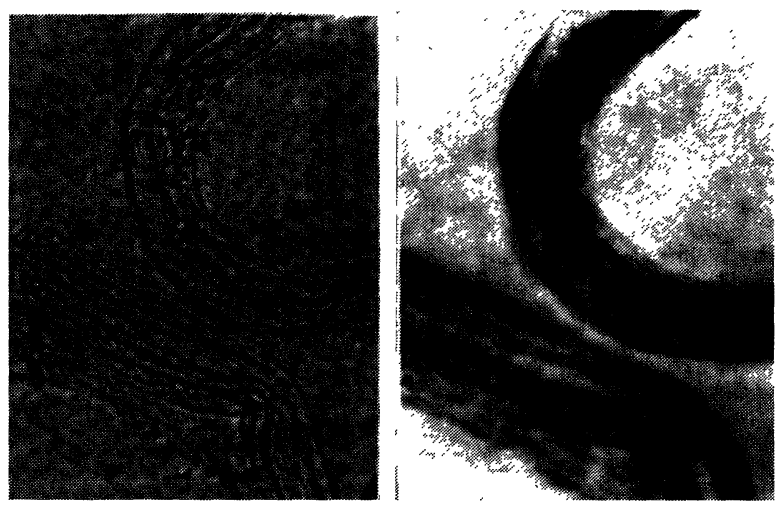

FIG. 23. - Images d'une fibre nerveuse : $a$ ) optique, $b$ ) ultra sonore (600 MHz) (Lemons, Quate [38]).

3.3.2 Synthèse d'une ouverture focalisante par couplage avec une ligne à retard ultrasonore. - Une technique ingénieuse d'exploration à grande ouverture avec déplacement rapide est en cours de développement à l'Université de Stanford [39] et consiste à coupler électroniquement par des composants non linéaires, tels que des diodes, l'onde acoustique reçue au niveau d'une barrette linéaire de transducteurs avec une onde ultrasonore de surface propagée sur une ligne à retard disposée parallèlement à la barrette et modulée linéairement en fréquence. Au signal injecté dans cette ligne et modulé selon la loi $\omega=\omega_{1}+\mu t$, correspond un signal propagé dont la phase s'écrit après une propagation sur la distance $x$ notant $C_{\mathrm{S}}$ la vitesse de l'onde de surface

$$
\varphi_{\mathrm{S}}=\left[\omega_{1}+\mu\left(t-\frac{x}{C_{\mathrm{S}}}\right)\right]\left(t-\frac{x}{C_{\mathrm{S}}}\right)=\mu \frac{x^{2}}{C_{\mathrm{S}}^{2}}+\cdots
$$

Cette phase possède une dépendance spatiale identique à la signature de Fresnel d'une onde sphérique reçue par la barrette. Le couplage non linéaire effectué au niveau des transducteurs correspondants de la barrette et de la ligne à retard (Fig. 24) fournit un signal contenant des composantes spectrales en $\omega \pm \omega_{\mathrm{A}}, \omega_{\mathrm{A}}$ étant la pulsation ultrasonore utilisée pour l'imagerie. Naturellement le signal obtenu résultant de la somme (sur l'espace) des contributions fournies au niveau de chaque couple de transducteurs, ne sera important que si la phase associée à l'une de ces composantes $\varphi_{\mathrm{A}} \pm \varphi_{\mathrm{S}}$ est spatialement stationnaire. Le réglage adéquat du paramètre $\mu$ de modulation de fréquence permet ainsi de sélectionner l'information acoustique, 


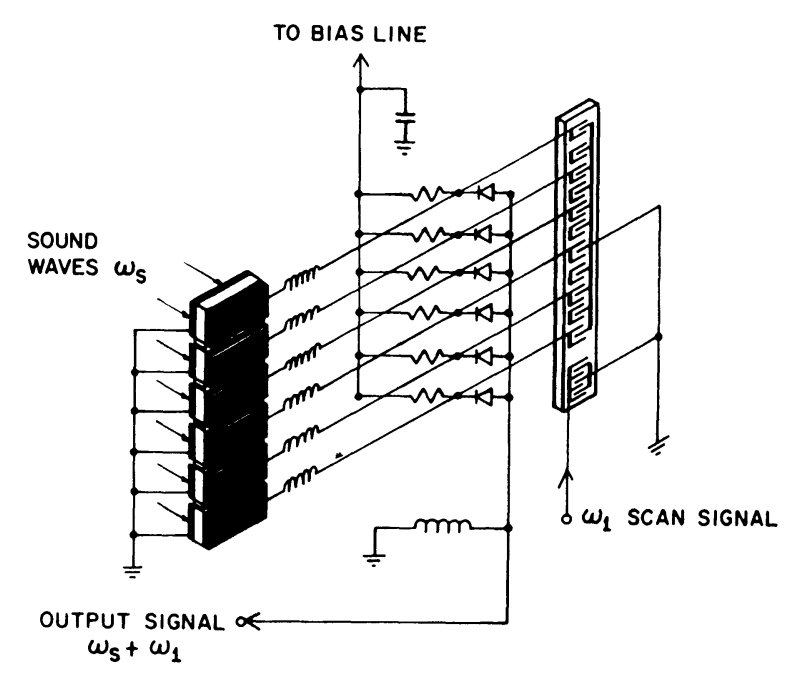

FIG. 24. - Imagerie par couplage non linéaire à une ligne à retard à ondes de surface (Havlice et al. [39]).

délivrée par une source ponctuelle à la distance $d$ (telle que par identification du terme quadratique en $x$ :

$$
\left.\frac{\pi}{\lambda_{\mathrm{A}} d}=\frac{\mu}{C_{\mathrm{S}}^{2}} \quad \text { soit } \quad d=\frac{\pi C_{\mathrm{S}}^{2}}{\lambda_{\mathrm{A}} \mu}\right),
$$

et localisée et émise de manière à ce que sa signature au niveau de la barrette soit centrée comme la signature $\varphi_{\mathrm{S}}$ à l'instant $t$. Cette dernière se propageant sur la ligne à retard avec la célérité $C_{\mathrm{S}}$, se comporte donc en gros comme une lentille focalisante qu'on déplacerait à la même vitesse selon la direction $x$. L'inconvénient de ce procédé est qu'il concerne une focalisation seulement bidimensionnelle, équivalente à une lentille cylindrique et non sphérique ne sélectionnant pas l'information selon la direction perpendiculaire à $x$. On peut remédier à cela en utilisant le même procédé à l'émission pour illuminer sélectivement la ligne $y=$ Cte de l'objet exploré très rapidement par le dispositif précédent ce qui permet alors une exploration séquentielle en $y$, sauvegardant le temps réel.

3.3.3 Synthèse et balayage électroniques d'une ouverture focalisante. - Naturellement la correction quadratique de phase obtenue par couplage à une ligne à retard dans le dispositif précédent revient à effectuer la transformation de Fresnel inverse requise pour restituer le plan objet à une distance donnée $d$ de la pupille de réception, tout au moins selon la direction $x$ de la barrette. On peut aussi apporter une telle correction de phase à l'information délivrée par les transducteurs d'un réseau matriciel par des moyens purement électroniques à l'aide de circuits de commutation et de déphasage, et synthétiser une ouverture focalisante dont on peut ainsi varier électroniquement les dimensions, la position, la distance focale, etc... Cependant la complexité de l'électronique à mettre en œuvre limite à ce jour ce genre de réalisation à des barrettes unidimensionnelles plutôt adaptées à l'exploration rapide de coupes échographiques de type B. L'exploration du plan de coupe peut être effectuée soit à partir d'une ouverture fixe avec un déphasage à l'émission des éléments autorisant un balayage sectoriel de la direction de l'émission ultrasonore comme pour les radars à balayage électronique $[40,41]$ soit à partir d'une ouverture émettant normalement et déplacée le long de la barrette par commutation soit encore par une combinaison des deux procédés [42]. Dans tous les cas la résolution peut être obtenue par une focalisation à la réception qui est adaptée électroniquement, à mesure que les échos parviennent, à la distance, croissante linéairement avec le temps, de leur origine. Une complexité raisonnable limite le nombre des transducteurs utilisés pour synthétiser l'ouverture à la vingtaine.

Une autre technique électronique permettant d'envisager un plus grand nombre de transducteurs dans une barrette ou même dans un réseau matriciel sans complexité prohibitive [43] consiste à effectuer une discrétisation binaire de la phase selon l'échantillonnage $(0, \pi)$ et à remplacer la signature de phase correctrice en

$$
\exp \left(j \pi \frac{(m p)^{2}}{\lambda_{\mathrm{A}} z}\right)
$$

au niveau du point $P$ de la pupille pour atteindre la source $\mathbf{M}\left(\mathbf{m}, z_{\mathbf{M}}\right)$ par la fonction $\Pi\left(\frac{\pi(m p)^{2}}{\lambda_{\mathbf{A}} z_{\mathbf{M}}}\right)$ définie par

$$
\begin{aligned}
\Pi(\varphi) & =+1 \quad \text { si } \quad-\frac{\pi}{2}<\varphi \leqslant \frac{\pi}{2}(2 \pi) \\
& =-1 \quad \text { si } \frac{\pi}{2}<\varphi \leqslant \frac{3 \pi}{2}(2 \pi)
\end{aligned}
$$

ce qui revient à doter chaque transducteur d'un simple inverseur électronique permettant d'apporter sa contribution en phase ou en antiphase au signal de réception. Ce procédé apporte apparemment une ambiguïté gênante dans l'imagerie puisque la décomposition de $\Pi(\varphi)$ en somme de $\mathrm{e}^{j n \varphi}$ ( $n$ entier $\gtrless 0$ ) fait apparaître une imagerie simultanée de sources disposées en $z= \pm z_{n} / n$ phénomène bien connu en optique où on sait qu'un réseau de Soret, dont la transparence s'écrit

$$
t(r)=\frac{1}{2}\left(1+\Pi\left(\frac{\pi r^{2}}{\lambda d}\right)\right)
$$

constitue une lentille multifocale. En fait l'utilisation d'un tel procédé de focalisation en échographie minimise considérablement cette ambiguité puisque les foyers parasites n'apportent pas de contribution importante pendant le temps utile de réception. Par ailleurs, l'émission pulsée utilisée en échographie possède un spectre assez large autour de la fréquence centrale ce qui fait qu'au lieu de focaliser selon une tache focale bien définie, on peut dans ces conditions obtenir une bonne résolution latérale pour tous les points situés sur un segment de part et d'autre de la tache focale (Fig. 25), d'autant plus allongé que le spectre d'émission est plus large, ce qui confère au dispositif une appréciable profondeur de champ. Une 

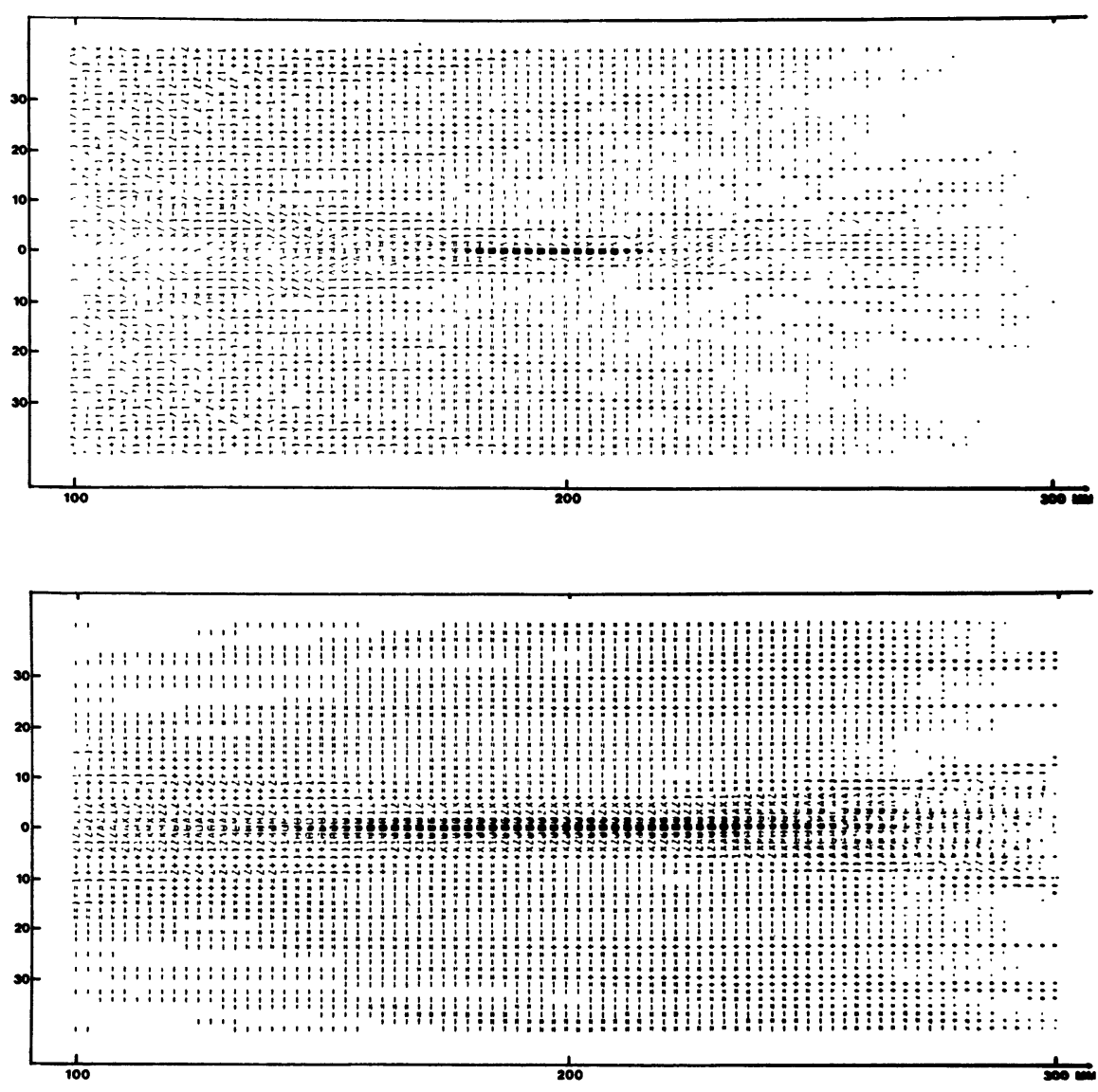

Fig. 25. - Diagramme de rayonnement (calcul numérique) d'un réseau linéaire de transducteurs excités en phase ou en antiphase : a) par un signal quasi monochromatique ( $>10$ périodes), $b$ ) par un signal à large spectre ( 2 périodes).

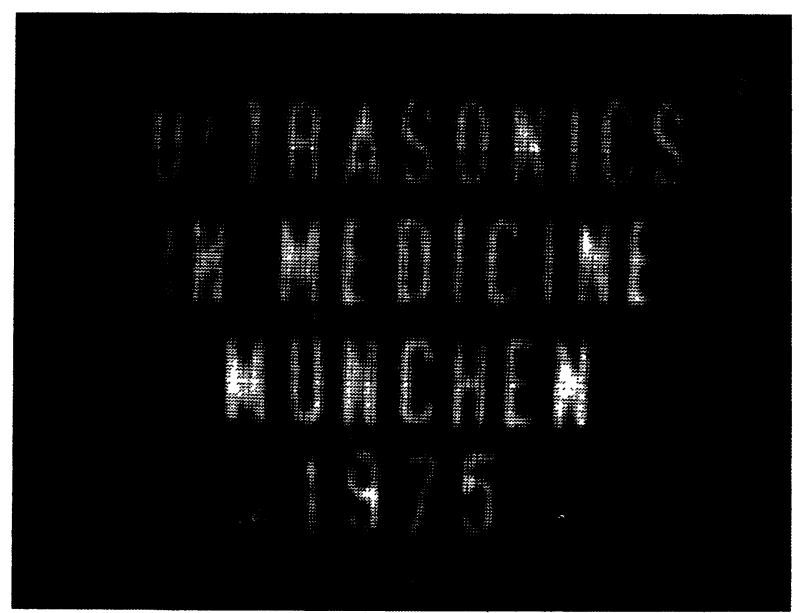

FIG. 26. - Echographie (obtenue à partir de lettres de plastique [43]).

barrette de 160 éléments utilisée de cette façon a ainsi permis d'obtenir à 2 et $3 \mathrm{MHz}$ des échographies $\mathrm{B}$ à la cadence de 16 images/s pour 160 lignes utilisées et des images d'échographie C (Fig. 26), en $15 \mathrm{~s}$ approximativement, en utilisant une ouverture synthétique pouvant regrouper jusqu'à 64 transducteurs. Une technique similaire bidimensionnelle est en projet utilisant la rétine électrostatique décrite en 3.2.4 avec une polarisation de ligne effectuée positivement ou négativement selon un groupement de Fresnel analogue à celui adopté pour la connexion en phase ou en antiphase dans la barrette précédente lequel est utilisé cette fois pour les colonnes.

4. Conclusion. - La présentation qui a été effectuée plus haut des techniques d'holographie ou d'imagerie acoustique présente d'inévitables arbitraires et est loin d'être complète. Après la relative déception éprouvée à la suite des résultats insuffisants pour leur application effective des premières techniques d'holographie acoustique les nombreux chercheurs acquis à l'intérêt de l'imagerie acoustique se tournent de plus en plus vers des dispositifs s'inspirant des classiques appareils d'échographie dont l'usage est imposé pour le diagnostic médical, afin d'améliorer les performances de ces derniers en rapidité par le balayage électronique et en résolution par la synthèse de grandes ouvertures. L'évolution très rapide des techniques électroniques et l'évidence pour une large audience de physiciens, de médecins et d'industriels, de l'intérêt du problème, permet d'espérer le développement à moyen terme d'appareils raffinés permettant d'obtenir non pas une image mais des données ultrasonores tridimensionnelles observables sous des angles différents, soit exactement l'information que l'holographie acoustique devrait en principe fournir, mais qui sera obtenue probablement d'une manière assez différente. Par 
ailleurs les techniques Doppler permettant de sélectionner les échos renvoyés par les cibles mobiles que sont les globules sanguins associés aux techniques d'imagerie devraient permettre la visualisation en volume de la vascularisation. Il n'a pas été du tout question des techniques d'imagerie sous-marine qui, du fait des vitesses relatives importantes de la cible et du dispositif de visualisation, requièrent le traitement en parallèle des informations échographiques cohérentes reçues sur un grand nombre de transducteurs répartis dans l'ouverture soit selon un réseau matriciel, soit de façon pseudo-aléatoire et font appel de plus en plus à un traitement numérique de l'information effectué en temps réel ou très peu différé par des micro-ordinateurs spécifiques [44]. Un autre grand espoir est attaché à l'amélioration de la prospection géophysique utilisant un vaitement similaire de l'information obtenue à partir de géophones répartis judicieusement à la surface du zol $[45,46,47]$.

Annexe 1. - Détermination Classique DES EFFETS INDUITS PAR LA LIMITATION D'OUVERTURE. - Remontant dans l'espace et dans le temps, ce qui est toujours facile en mathématiques, du plan $\pi_{z_{\mathbf{I}}}$ à celui de la pupille $\pi_{0}$ on peut écrire l'onde émergente en termes de l'image idéale $U_{\mathrm{I}}(\mathbf{i})$

$U_{+}(\mathbf{p})=-\frac{1}{j \lambda_{0} z_{\mathrm{I}}} \int U_{\mathrm{I}}(\mathbf{i}) \exp \left(-j \pi \frac{(i p)^{2}}{\lambda_{0} z_{\mathrm{I}}}\right) \mathrm{d} S_{\mathrm{I}}$,

ce qui permet d'étudier les perturbations apportées par le découpage de la pupille défini par la fonction $P(\mathbf{p})$. En effet, si on revient à nouveau dans le plan-image $\pi_{z_{\mathrm{I}}}$ en tenant compte de la géométrie de la pupille, le rayonnement qu'on y observe en fait doit s'écrire :

$$
\begin{aligned}
& U_{z_{\mathrm{I}}}\left(\mathbf{m}_{\mathrm{I}}\right)= \frac{1}{j \lambda_{0} z_{\mathrm{I}}} \int P(\mathbf{p}) U_{+}(\mathbf{p}) \exp \left(j \pi \frac{\left(p m_{\mathrm{I}}\right)^{2}}{\lambda_{0} z_{\mathrm{I}}}\right) \mathrm{d} S_{\mathrm{P}} \\
&= \frac{1}{\lambda_{0} z_{\mathrm{I}}^{2}} \int \mathrm{d} S_{\mathrm{I}} U_{\mathrm{I}}(\mathbf{i}) \int \mathrm{d} S_{\mathrm{P}} P(\mathrm{i} ; \times \\
& \quad \times \exp \left(j \pi \frac{\left(p m_{\mathrm{I}}\right)^{2}-(p i)^{2}}{\lambda_{0} z_{\mathrm{I}}}\right) \\
&=\frac{1}{\lambda_{0} z_{\mathrm{I}}^{2}} \int \mathrm{d} S_{\mathrm{I}} U_{\mathrm{I}}(\mathbf{i}) \exp \left(j \pi \frac{m_{\mathrm{I}}^{2}-i^{2}}{\lambda_{0} z_{\mathrm{I}}}\right) \times \\
& \quad \times \int \mathrm{d} S_{\mathrm{P}} P(\mathbf{p}) \exp \left(-2 j \pi \frac{\mathbf{p} \cdot i \mathbf{m}_{\mathrm{I}}}{\lambda_{0} z_{\mathrm{I}}}\right)
\end{aligned}
$$

et, notant $A_{\mathrm{P}}(\mathbf{f})$ la transformée de Fourier de la fonction pupille $P(\mathbf{p})$

$$
\begin{array}{r}
U_{z_{\mathbf{I}}}\left(\mathbf{m}_{\mathrm{I}}\right)=\frac{1}{\lambda_{0}^{2} z_{\mathrm{I}}^{2}} \int \mathrm{d} S_{\mathrm{I}} U_{\mathrm{I}}(\mathbf{i}) \exp \left(j \pi \frac{m_{\mathrm{I}}^{2}-i^{2}}{\lambda_{0} z_{\mathrm{I}}}\right) \times \\
\times A_{\mathrm{P}}\left(\frac{\mathbf{i} \mathbf{m}_{\mathrm{I}}}{\lambda_{0} z_{\mathrm{I}}}\right) .
\end{array}
$$

On vérifie que, si la longueur d'onde $\lambda_{0}$ est suffisam- ment petite pour que la pupille puisse être considérée comme infiniment grande, la fonction $A_{\mathrm{P}}$ devient une distribution ponctuelle

$$
\begin{aligned}
A_{\mathrm{P}}\left(\frac{\mathbf{i} \mathbf{m}_{\mathrm{I}}}{\lambda_{0} z_{\mathrm{I}}}\right) & =\delta\left(\frac{\mathbf{i} \mathbf{m}_{\mathrm{I}}}{\lambda_{0} z_{\mathrm{I}}}\right) \\
U_{z_{\mathrm{I}}}\left(\mathbf{m}_{\mathrm{I}}\right) & =\frac{1}{\lambda_{0}^{2} z_{\mathrm{I}}^{2}} U_{\mathrm{I}}\left(\mathbf{m}_{\mathrm{I}}\right) * \delta\left(\frac{\mathbf{m}_{\mathrm{I}}}{\lambda_{0} z_{\mathrm{I}}}\right)=U_{\mathrm{I}}\left(\mathbf{m}_{\mathrm{I}}\right) .
\end{aligned}
$$

Si les dimensions finies de la pupille apparaissent, la fonction

$$
h\left(\mathbf{i m}_{\mathrm{I}}\right)=\frac{1}{\lambda_{0}^{2} z_{\mathrm{I}}^{2}} A_{\mathrm{P}}\left(\frac{\mathbf{i m}_{\mathrm{I}}}{\lambda_{0} z_{\mathrm{I}}}\right) .
$$

reste cependant distribuée essentiellement au voisinage immédiat de $\mathbf{i}$ en sorte qu'on peut négliger la variation de phase en $\pi \frac{m_{\mathrm{I}}^{2}-i^{2}}{\lambda_{0} z_{\mathrm{I}}}$ dans ce voisinage et continuer d'écrire l'onde image en terme du produit de convolution :

$$
U_{z_{\mathbf{I}}}\left(\mathbf{m}_{\mathbf{I}}\right)=U_{\mathbf{I}}\left(\mathbf{m}_{\mathbf{I}}\right) * h\left(\mathbf{m}_{\mathbf{I}}\right)
$$

Annexe 2. - EFFETS INDUITS PAR ÉCHANTILlONNAGE INTÉGRÉ SUR UN CRÉNEAU. - En pratique l'échantillonnage est effectué par un transducteur de surface non nulle qui fournit l'information moyennée sur cette surface en sorte que si on note par exemple

$$
\begin{aligned}
& \Pi\left(\mathbf{p} / \delta^{\prime}\right)=1 \text { si }\left|p_{x}\right| \text { et }\left|p_{y}\right|<\delta^{\prime} / 2 \\
& =0 \text { autrement, }
\end{aligned}
$$

le réseau de transducteurs à surface carrée de côté $\delta^{\prime}$ centrés sur le maillage de pas $\delta$ défini précédemment transmet l'information selon

$$
U_{+}=U_{-} P(\mathbf{p}) \operatorname{Pgn}(\mathbf{p} / \delta) * \frac{1}{\delta^{\prime 2}} \Pi\left(\mathbf{p} / \delta^{\prime}\right),
$$

où l'opération de moyenne qui se traduit par une corrélation est ici écrite selon un produit de convolution, ce qui est justifié du fait de la symétrie en p de la fonction $\Pi(\mathbf{p})$. On peut alors atteindre l'image $U_{z_{\mathbf{I}}}(\mathbf{m})$ selon

$$
\begin{aligned}
U_{z_{\mathrm{I}}}(\mathbf{m})=U_{-}(\mathbf{m}) P(\mathbf{m}) \operatorname{Pgn}\left(\frac{\mathbf{m}}{\delta}\right) & * \frac{1}{\delta^{\prime 2}} \Pi\left(\frac{\mathbf{m}}{\delta}\right) * \frac{1}{j \lambda_{0} z_{\mathrm{I}}} \times \\
& \times \exp \left(j \pi \frac{m^{2}}{\lambda_{0} z_{\mathrm{I}}}\right)
\end{aligned}
$$

le dernier produit de convolution traduisant la propagation de $\pi_{0}$ à $\pi_{z_{\mathbf{I}}}$

Il est avantageux de commuter les deux opérations de convolution dans l'expression (41), ce qui permet d'interpréter l'effet de moyenne spatiale opéré par les transducteurs par une opération de lissage de l'image définie en (38), (27') selon

$$
U_{z_{\mathbf{I}}}^{\prime \prime}(\mathbf{m})=U_{z_{\mathbf{I}}}^{\prime}(\mathbf{m}) * \frac{1}{\delta^{\prime 2}} \Pi\left(\mathbf{m} / \delta^{\prime}\right)
$$


Cette opération de lissage peut avoir un effet tout à fait bénéfique dans la mesure où elle conduit, au prix d'un certain élargissement de la tache centrale, à une apodisation de l'image d'ordre zéro et à une réduction considérable des images d'ordre n. A cet égard, l'effet de moyenne est identique à l'apodisation qu'on peut effectuer comme il a été dit plus haut en modulant la sensibilité de la pupille par une fonction atténuant les effets du découpage brutal et revient dans le cas présent à moduler la pupille selon la loi

$$
P^{\prime}(\mathbf{p})=P(\mathbf{p}) \mathcal{A}(\mathbf{p}) .
$$

En effet, si on note

$$
L(\mathbf{m})=\frac{1}{\delta^{\prime 2}} \Pi\left(\frac{\mathbf{m}}{\delta^{\prime}}\right),
$$

la fonction de lissage, la réponse impulsionnelle lissée :

$$
h^{\prime}=h * L
$$

est bien associée en vertu de (32) à la pupille :

$$
P^{\prime}(\mathbf{p})=P(\mathbf{p}) A_{L}\left(\frac{-\mathbf{p}}{\lambda_{0} z_{\mathrm{I}}}\right)
$$

ce qui permet d'expliciter la fonction atténuatrice :

$$
\begin{aligned}
\mathcal{A}(\mathbf{p}) & =A_{L}\left(\frac{-\mathbf{p}}{\lambda_{0} z_{\mathrm{I}}}\right) \\
& =\frac{\sin \pi \frac{\delta^{\prime}}{\lambda_{0} z_{\mathrm{I}}} p_{x}}{\pi \frac{\delta^{\prime}}{\lambda_{0} z_{\mathrm{I}}} p_{x}} \frac{\sin \pi \frac{\delta^{\prime}}{\lambda_{0} z_{\mathrm{I}}} p_{y}}{\pi \frac{\delta^{\prime}}{\lambda_{0} z_{\mathrm{I}}} p_{y}} .
\end{aligned}
$$

\section{Bibliographie}

[1] Langevin, P. et Chilowsky, C., Procédés et Appareils pour la production de signaux dirigés et pour la localisation d'obstacles sous-marins, Brevet français $\mathrm{n}^{\circ} 502913$ du 29 mai 1916.

[2] Duffieux, P. M., L'Intégrale de Fourier et ses applications à l'optique, Faculté des Sciences, Besançon 1946.

[3] Maréchal, A., Croce, P., Hebd. Séan. Acad. Sci. 237 (1953) 667.

[4] Lowenthal, S., Belvaux, Y., Progrès récents en optique cohérente : filtrage des fréquences spatiales, holographie, Rev. Opt. Théor. Inst. 46 (1967) 1-64.

[5] Goodman, J., Introduction to Fourier Optics.

[6] GABOR, D., Microscopy by Reconstructed Wavefronts. (I) Proc. Phys. Soc. A 197 (1959) 454.

(II) Proc. Phys. Soc. B 64 (1951) 449.

[7] Sokoloff, S., Means for Indicating Flaws in Materials, U. S. S. R. Patent no 49 (1936), U. S. Patent no 2164125 (1939).

[8] Oschepkov, P. K., Rosenberg, L. D., Semennikov, Yu. B., Electroacoustic converter for the visualisation of acoustic images, Sov. Phys. Acoust. 1 (1955) 362-391.

[9] SMYth, C. N., SAYers, J. F., Ultrasonic Image Converter, The Engineer 207 (1959) 348.

[10] Jacobs, J. E., Berger, H., Collis, W. J., An Investigation of the limitations to the maximum attainable sensitivity in Acoustical Image Converters, I.E.E.E. Trans. Ultrasonics Eng. U. E. 10 (2), 83-88 (1963).

[11] MarinI, J., Etude de l'émission électronique secondaire de quelques matériaux piézoélectriques. Applications à la visualisation ultrasonore, Thèse, Fac. des Sci. Lyon, 1969.

[12] Dubors, J. L., Large Aperture Acoustical Image Converters, Acoust. Holog. 2 (1970) 59-68.

[13] Jacobs, J. E., Peterson, D. A., Advances in the Sokoloff Tube, Acoust. Holog. 5 (1974) 633-645.

[14] Green, P. S., Schaefer, L. F., Jones, P. D., Suarez (J. R.), A New High Performance Ultrasonic camera, Acoust. Holog. 5 (1974) 493-503.

[15] $\mathrm{VI}^{\mathrm{e}}$ International Congress on Acoustical Holography and Imaging, fev. 75, San Diego.

[16] Metherell, A. F., The Relative Importance of Phase and Amplitude, Acoust. Holog. 1 (1969) 203-221.

[17] Mueller, R. K., Sheridon, N. K., Sound Holograms and Optical Reconstruction, Appl. Phys. Lett. 9 (1966) 328.

[18] Mueller, R. K., Keating, P. N., The Liquid-Gas Interface as a Recording Medium for Acoustical Holography, Acoust. Holog. 1 (1969) 49-56.
[19] Mueller, R. K., Acoustic Holography, Proc. of the I. E. E. E. 59 (1971) 1319-1335.

[20] SMith, R. B., BRENDEN, B. B., Refinements and Variations in Liquid Surface and Scanned Ultrasound Holography, I. E. E. E., Trans Sonics Ultras. SV 16 (1969) 29.

[21] Metherell, A. F., SpinaK, S., Pisa, E. J., Temporal Reference Acoustical Holography, Acoust. Holog. 2 (1970) 69-85.

[22] Mezrich, R., Etzold, K., Vilkomerson, D., Ultrasonovision, Communication $\mathrm{VI}^{\mathrm{e}}$ Congrès Acoustical Holog. and Imaging San Diego Fev. 1975.

[23] Korpel, A., Desmares, P., Rapid Sampling of Acoustical Holograms by Laser Scanning Techniques, J. Opt. Soc. Amer. 45 (1969) 881.

[24] Kessler, L. W., Palermo, P. R., Korpel, A., Recent Developments with Scanning laser Acoustic Microscope, Acoust. Holog. 5 (1974), 15-23.

[25] Gabor, D., A Project of Ultrasonic Tomography (Sonoradiography), Ultrasonic Imaging and Holography (G. W. Stroke et al., edit.) 1974, p. 151-158.

[26] Takgi, N., Kawashima, T., Ogura, T., Yamada, T., Solid State Acoustic Image Sensor, Acoust. Holog. 4 (1972) 215-236.

[27] Maginness, M. G., Plummer, J. D., Meinde, J. D., An Acoustic Image Sensor Using a Transmit Receive Array, Acoust. Holog. 5 (1974) 619-631.

[28] AlAIs, P., Acoustical Imaging by Electrostatic Transducers, Acoust. Holog. 4 (1972) 237-250.

[29] AlAis, P., Real Time Acoustical Imaging with a $256 \times 256$ Matrix of Electrostatic Transducers, Acoust. Holog. 5 (1974) 671-684.

[30] Korpel, A., Visualization of the Cross Section of Sound Beam by Bragg Diffraction of Light, Appl. Phys. Lett. 9 (1966) 425.

[31] LANDRY, J., Powers, J., Wade, G., Ultrasonic Imaging of Internal Structure by Bragg Diffractions, Appl. Phys Lett. 15 (1969) 186.

[32] Landry, J., Smith, R., Wade, G., Optical Detection in Bragg Imaging, Acoust. Holog. 3 (1972) 47-69.

[33] BaERD, B., Guinot, J. C., Dispositif de visualisation ultrasonore par diffraction de Bragg, Communication Congrès Français de Mécanique, sept. 75, Toulouse.

[34] Green, P. S., Schaefer, L. F., Macovski, A., Considerations for Diagnostic Ultrasonic Imaging, Acoust. Holog. 4 (1972) $97-111$ 
[35] Aldridge, E. E., Clare, A. B., Shepherd, D. A., Scanned Ultrasonic Holography for Ophtalmic Diagnosis, Ultrasonics 12 (1974) 155-160.

[36] Havlice, J. F., Quate, C. F., Goodman, J. W., Acoustic microscope vizualization of Acoustic Beams, Electr. Res. Rev. 11 (1969).

[37] Auld (B. A.), Gilbert (P. J.), Hyllested (K.), Roberts (C. G.), WebB, D. C., A 1. 1. Ghz. Scanned Acoustic Microscope.

[38] Lemons, R. A., Quate, C. F., Acoustic Microscopy. A Tool for Medical and Biological Research, Communication $\mathrm{VI}^{\mathrm{e}}$ Congrès Acoustical Holog. and Imaging, fév. 75 San Diego.

[39] Havlice, J. F., Kino, G. S., Kofol, J. S., Quate, C. F., An Electronically Focused Acoustic Imaging Device, Acoust. Holog. 5 (1974) 317-333.

[40] Somer, J. C., Electronic Sector Scanning with Ultrasonics Beams, Proc. of the $1^{\mathrm{er}}$ Congress on Ultrasonics in Medicine, June 1969, Vienne.

[41] Thurstone, F. L., Ramm, O. T., A New Ultrasound Imaging Technique Employing two dimensional Electronic Beam steering, Acoust. Holog. 5 (1974) 249-259.
[42] Pourcelot, L., Pottier, J. M., Berson, M., Planiol, Th., Fast Ultrasonic Imaging System (U. S. A. B. E. L.), Communication Congrès "Ultrasonics in Medicine " mai 75, Munich.

[43] Alais, P., Fink, M., Perrin, J., Acoustic Imaging with an Electronically Focused and Scanned Array, Communication Congres "Ultrasonics in Medicine», Mai 75, Munich.

[44] Keating, P. N., Koppelmann, R. F., Mueller, R. F., Steinberg, R. F., Complex on Axis. Holograms and reconstruction without conjugate images, Acoust. Holog. $5,515-526$

[45] Fontanel, A., Grau, G., Holographies monochromatique et impulsionnelle, Communication Symposium International sur les Applications de 1'Holographie, juillet 1970, Besançon.

[46] Fizpatrick, G. L., First Arrivel Seismic Holograms, Acoust. Holog. 4 (1972) 381-400.

[47] FARR, J. B., Earth Holography as a Method to delineate buried structures, Communication $\mathrm{VI}^{\mathrm{e}}$ Congress of Acoustical Holography and Imaging, Feb. 75, San Diego. 